\title{
Quantum Broadcast Channels with Cooperating Decoders: An Information-Theoretic Perspective on Quantum Repeaters
}

\author{
Uzi Pereg, ${ }^{1, a)}$ Christian Deppe, ${ }^{1, b)}$ and Holger Boche ${ }^{2,3, c)}$ \\ 1) Institute of Communication Engineering, Technical University of Munich. \\ ${ }^{2)}$ Theoretical Information Technology, Technical University of Munich. \\ ${ }^{3)}$ Munich Center for Quantum Science and Technology (MCQST). ${ }^{\mathrm{d})}$
}

(Dated: 18 June 2021)

Communication over a quantum broadcast channel with cooperation between the receivers is considered. The first form of cooperation addressed is classical conferencing, where Receiver 1 can send classical messages to Receiver 2. Another cooperation setting involves quantum conferencing, where Receiver 1 can teleport a quantum state to Receiver 2. When Receiver 1 is not required to recover information and its sole purpose is to help the transmission to Receiver 2, the model reduces to the quantum primitive relay channel. The quantum conferencing setting is intimately related to quantum repeaters, as the sender, Receiver 1, and Receiver 2 can be viewed as the transmitter, the repeater, and the destination receiver, respectively. We develop lower and upper bounds on the capacity region in each setting. In particular, the cutset upper bound and the decode-forward lower bound are derived for the primitive relay channel. Furthermore, we present an entanglementformation lower bound, where a virtual channel is simulated through the conference link. At last, we show that as opposed to the multiple access channel with entangled encoders, entanglement between decoders does not increase the classical communication rates for the broadcast dual.

Keywords: Quantum communication, Shannon theory, broadcast channel, conferencing, quantum repeater.

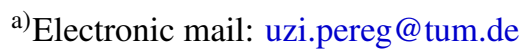

b)Electronic mail: christian.deppe@tum.de

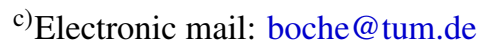

${ }^{d)}$ Part of this work has been presented at the IEEE International Symposium on Information Theory (ISIT 2021), Melbourne, Australia, July 12 - 20, 2021.
} 


\section{INTRODUCTION}

Attenuation in optical fibers poses a great challenge for long-distance quantum communication protocols, including both current applications such as quantum key distribution ${ }^{1}$, as well as future implementation of the quantum internet ${ }^{2}$ and quantum networks in general ${ }^{3}$. Quantum repeaters have been proposed as a potential solution where the distance is divided into smaller segments with quantum repeaters at the intermediate stations ${ }^{4}$. In its simplest form, the process begins with using quantum communication and entanglement distillation to prepare two pairs of qubits at maximally entangled states, namely, $\left|\Phi_{A P_{1}}\right\rangle$ between the sender and the repeater, and $\left|\Phi_{P_{2} B}\right\rangle$ between the repeater and the receiver. At the next stage, the repeater teleports the quantum state of $P_{1}$ onto $B$ thus swapping the entanglement such that $A$ and $B$ are now entangled at a distance twice that of the initial entangled pairs. Experimental implementation of the elementary building blocks for quantum repeaters has recently been considered by van Loock et al. ${ }^{5}$ in platforms based on

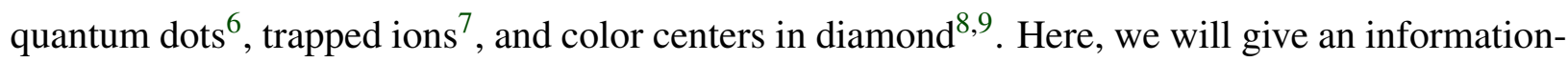
theoretic perspective that can be associated with such a network.

The cross-disciplinary field of quantum information processing and communication is rapidly evolving in both practice and theory ${ }^{10-17}$. Quantum information theory is the natural extension of the classical theory. Nevertheless, this generalization reveals astonishing phenomena with no parallel in classical communication ${ }^{18}$. For example, pairing two memoryless quantum channels, each with zero quantum capacity, can result in a nonzero quantum capacity ${ }^{19}$. This property is referred to as super-activation. It should be noted that super-activation has also been demonstrated in recent years for classical channels in advanced settings, such as secure message transmission over a wiretap channel with a jammer ${ }^{20}$ and identification over a discrete memoryless channel with feedback $^{21}$. Nevertheless, super-activation does not occur in the fundamental model of a classical one-way memoryless channel.

Communication over quantum channels can be separated into different tasks and categories. For classical information transmission, a regularized ("multi-letter") formula for the capacity of a quantum channel without assistance was established by Holevo ${ }^{22}$, and Schumacher et al. ${ }^{23}$. Although the calculation of such a formula is intractable in general, it provides computable lower bounds, and there are special cases where the capacity can be computed exactly. The reason for this difficulty is that the Holevo information is not necessarily additive ${ }^{24}$. A similar difficulty occurs with the transmission of quantum information. A regularized formula for the quantum 
capacity is given in Refs. 25-28. A computable formula is obtained in the special case where the channel is degradable ${ }^{29}$, or belongs to the more general class of less noisy channels ${ }^{30}$. Quantum communication can also be used for the purpose of entanglement generation ${ }^{28,31,32}$.

Another scenario of interest is when the transmitters and receivers are provided with entanglement resources a priori ${ }^{33-35}$. While entanglement can be used to produce shared randomness, it is a much more powerful aid ${ }^{3,36,37}$. In particular, super-dense coding ${ }^{38}$ is a well-known communication protocol where two classical bits are transmitted using a single use of a noiseless qubit channel and a maximally entangled pair that is shared between the transmitter and the receiver. Thereby, transmitter-receiver entanglement assistance doubles the transmission rate of classical messages over a noiseless qubit channel. The entanglement-assisted capacity of a noisy quantum channel was fully characterized by Bennet et al. ${ }^{39,40}$ in terms of the quantum mutual information. In the other direction, i.e. using information measures to understand quantum physics, the quantum mutual information plays a role in investigating the entanglement structure of quantum field theories ${ }^{41-44}$.

There are communication settings where entanglement resources can even increase the capacity of a classical channel. In particular, Leditzky et al. ${ }^{45}$ have recently shown that entanglement between two transmitters can strictly increase the achievable rates for a classical multiple access channel. The channel construction in Ref. 45 is based on a pseudo-telepathy game ${ }^{46}$ where quantum strategies guarantee a certain win and outperform classical strategies, extending ideas by Nötzel ${ }^{47}$ and Quek and Shor ${ }^{48}$. Entanglement assistance has striking effects in different communication games and their security applications as well ${ }^{45,49-53}$. Furthermore, entanglement can assist in the transmission of quantum information. Given a classical channel with transmitterreceiver entanglement resources, qubits can be sent at half the rate of classical bits by employing the teleportation protocol $^{54}$.

Quantum broadcast and multiple access channels were studied in various settings, as e.g. in Refs. 55-70 and 71-78. Yard et al. ${ }^{55}$ derived the superposition inner bound and determined the capacity region for the degraded classical-quantum broadcast channel. By the monogamy property of quantum entanglement ${ }^{79}$, the sender's system cannot be in a maximally entangled state with both receivers simultaneously. However, different forms of entanglement can be generated. In particular, Yard et al. ${ }^{55}$ characterize the entanglement-generation rates for GHZ states. Wang et $a l .{ }^{58}$ used the previous characterization to determine the capacity region for Hadamard broadcast channels. Dupuis et al. ${ }^{59,60}$ developed the entanglement-assisted version of Marton's region for 
users with independent messages. Bosonic broadcast channels are considered in Refs. 80-84. The quantum broadcast and multiple access channels with confidential messages were recently considered in Refs. 85-86 and 87-88, respectively. An equivalent description of the super-activation phenomenon ${ }^{19}$ is that there exists a broadcast channel such that the sum-rate capacity with full cooperation between the receivers is positive while the capacities of the marginal channels are both zero.

Savov et al. ${ }^{89,90}$ derived a partial decode-forward lower bound for the (non-primitive) classicalquantum relay channel, where the relay encodes information in a strictly-causal manner. Recently, Ding et al. ${ }^{91}$ generalized those results and established the cutset, multihop, and coherent multihop bounds for the classical-quantum relay channel. Communication with the help of environment measurement can be modelled by a quantum channel with a classical relay in the environment ${ }^{92}$. Considering this setting, Smolin et al. ${ }^{93}$ and Winter ${ }^{94}$ determined the environment-assisted quantum capacity and classical capacity, respectively. Savov et al. ${ }^{89}$ further discussed future research directions of interest (see Sec. V in Ref. 89), and pointed out that quantum communication scenarios over the relay channel may have applications for the design of quantum repeaters (see also Ref. 91). Our aim is to fulfill this prevision.

In this paper, we consider quantum broadcast channels in different settings of cooperation between the decoders. Using those settings, we provide an information-theoretic framework for quantum repeaters. The first form of cooperation that we consider is classical conferencing, where Receiver 1 can send classical messages to Receiver 2. This can be viewed as the quantum version of the classical setting by Dabora and Servetto ${ }^{95}$ (see also Ref. ${ }^{96}$ ). We provide a regularized characterization for the classical capacity region of the quantum broadcast channel with classical conferencing, and a single-letter formula for Hadamard broadcast channels ${ }^{58}$. Next, we consider quantum conferencing, where Receiver 1 can teleport a quantum state to Receiver 2. We develop inner and outer bounds on the quantum capacity region with quantum conferencing, characterizing the tradeoff between the communication rates $Q_{1}$ and $Q_{2}$ to Receiver 1 and Reciever 2, respectively, as well as the conferencing capacity $C_{Q, 12}$. The case where Receiver 1 is not required to recover information and its sole purpose is to help the transmission to Receiver 2, reduces to the model of the primitive relay channel ${ }^{97}$, for which the decode-forward lower bound and cutset upper bound follow as a consequence. In addition, we establish an entanglement-formation lower bound, where a virtual channel is simulated through the conference link, following the results of Berta et al. ${ }^{98}$ on quantum channel simulation. 
The quantum conferencing setting is intimately related to quantum repeaters, as the sender, Receiver 1, and Receiver 2 can be viewed as the transmitter, the repeater, and the destination receiver, respectively, in the repeater model. In particular, the sender can employ quantum communication to Receiver 1 (the repeater) in order to prepare a maximally entangled pair $\left|\Phi_{A P_{1}}\right\rangle$, which consists of $n Q_{1}$ entangled bits (ebits). Given entanglement between the receivers, we also have a maximally entangled pair $\left|\Phi_{P_{2} B}\right\rangle$, which consists of $n C_{Q, 12}$ ebits, shared between the repeater and the destination receiver. Then, the repeater can swap his entanglement by using the classical conferencing link to teleport the state of $P_{1}$ onto $B$ thus swapping the entanglement such that $A$ and $B$ are now entangled. Hence our results provide an information-theoretic analysis characterizing the achievable rates of ebits that can be generated in each stage. As our model includes direct transmission from $A$ to $B$ as well, our results exhibit the tradeoff between repeaterless communication and communication via the repeater. Other relay channel models for quantum repeaters can also be found in Refs. 99-103.

At last, we compare between entanglement cooperation for the multiple access channel and the broadcast channel. The duality between the multiple access channel and the broadcast channel has emerged as a prominent tool in the study of wireless communication systems ${ }^{104-106}$. We show that as opposed to the multiple access channel with entangled transmitters ${ }^{45}$, entanglement between the receivers cannot enlarge the classical capacity region of a broadcast channel. Furthermore, this property extends to any pair of non-signaling correlated resources that are shared between the receivers. Consequently, the broadcast dual to the multiple access channel property by Leditzky et al. ${ }^{45}$ does not hold. Hence, our result reveals a fundamental asymmetry and demonstrates the limitations of the duality between the broadcast channel and the multiple access channel.

The paper is organized as follows. In Sec. II, we begin with the basic definitions. In Sec. III, we present three coding scenarios for the quantum broadcast channel with cooperation between the receivers. In particular, we consider classical communication when the receivers share entanglement resources a priori (Subsec. III A); classical communication over the quantum broadcast channel with a classical conference link from Receiver 1 to Receiver 2 (Subsec. III B); and quantum communication when Receiver 1 can teleport a quantum state to Receiver 2 via conferencing (Subsec. III C). The quantum primitive relay channel is presented as a special case as well. Our results for classical conferencing are given in Sec. IV. Next, our main results on quantum conferencing are derived in Sec. V, for the quantum broadcast channel (V A,V B), and the quantum primitive relay channel (V C). Sec. V is concluded with the resulting observations on the quantum 
repeater. In Sec. VI, we show that the broadcast dual to the multiple access channel property by Leditzky et al. ${ }^{45}$ does not holds, as entanglement between receivers cannot enlarge the classical capacity region. We conclude with a summary and discussion in Sec. VII.

\section{DEFINITIONS}

\section{A. Notation, States, and Information Measures}

We use the following notation conventions. Script letters $\mathscr{X}, \mathscr{Y}, \mathscr{Z}, \ldots$ are used for finite sets. Lowercase letters $x, y, z, \ldots$ represent constants and values of classical random variables, and uppercase letters $X, Y, Z, \ldots$ represent classical random variables. The distribution of a random variable $X$ is specified by a probability mass function $(\mathrm{pmf}) p_{X}(x)$ over a finite set $\mathscr{X}$. We use $x^{j}=\left(x_{1}, x_{2}, \ldots, x_{j}\right)$ to denote a sequence of letters from $\mathscr{X}$. A random sequence $X^{n}$ and its distribution $p_{X^{n}}\left(x^{n}\right)$ are defined accordingly.

The state of a quantum system $A$ is a density operator $\rho$ on the Hilbert space $\mathscr{H}_{A}$. A density operator is an Hermitian, positive semidefinite operator, with unit trace, i.e. $\rho^{\dagger}=\rho, \rho \succeq 0$, and $\operatorname{Tr}(\rho)=1$. The state is said to be pure if $\rho=|\psi\rangle\langle\psi|$, for some vector $|\psi\rangle \in \mathscr{H}_{A}$, where $\langle\psi|$ is the Hermitian conjugate of $|\psi\rangle$. In general, a density operator has a spectral decomposition,

$$
\rho=\sum_{x \in \mathscr{X}} p_{X}(x)\left|\psi_{x}\right\rangle\left\langle\psi_{x}\right|
$$

where $\mathscr{X}=\left\{1,2, \ldots,\left|\mathscr{H}_{A}\right|\right\}, p_{X}(x)$ is a probability distribution over $\mathscr{X}$, and $\left\{\left|\psi_{x}\right\rangle\right\}_{x \in \mathscr{X}}$ forms an orthonormal basis of the Hilbert space $\mathscr{H}_{A}$. A measurement of a quantum system is any set of operators $\left\{\Lambda_{j}\right\}$ that forms a positive operator-valued measure (POVM), i.e. the operators are positive semi-definite and $\sum_{j} \Lambda_{j}=\mathbb{1}$, where $\mathbb{1}$ is the identity operator ${ }^{33}$. According to the Born rule, if the system is in state $\rho$, then the probability of the measurement outcome $j$ is given by $p_{A}(j)=\operatorname{Tr}\left(\Lambda_{j} \rho\right)$. The trace distance between two density operators $\rho$ and $\sigma$ is $\|\rho-\sigma\|_{1}$ where $\|F\|_{1}=\operatorname{Tr}\left(\sqrt{F^{\dagger} F}\right)$.

Define the quantum entropy of the density operator $\rho$ as $H(\rho) \triangleq-\operatorname{Tr}[\rho \log (\rho)]$, which is the same as the Shannon entropy associated with the eigenvalues of $\rho$. Consider the state of a pair of systems $A$ and $B$ on the tensor product $\mathscr{H}_{A} \otimes \mathscr{H}_{B}$ of the corresponding Hilbert spaces. Given a bipartite state $\sigma_{A B}$, define the quantum mutual information as

$$
I(A ; B)_{\sigma}=H\left(\sigma_{A}\right)+H\left(\sigma_{B}\right)-H\left(\sigma_{A B}\right) .
$$


Furthermore, conditional quantum entropy and mutual information are defined by $H(A \mid B)_{\sigma}=$ $H\left(\sigma_{A B}\right)-H\left(\sigma_{B}\right)$ and $I(A ; B \mid C)_{\sigma}=H(A \mid C)_{\sigma}+H(B \mid C)_{\sigma}-H(A, B \mid C)_{\sigma}$, respectively. The coherent information is then defined as

$$
I(A\rangle B)_{\sigma}=-H(A \mid B)_{\sigma}
$$

A pure bipartite state is called entangled if it cannot be expressed as the tensor product of two states in $\mathscr{H}_{A}$ and $\mathscr{H}_{B}$. The maximally entangled state between two systems of dimension $D$ is defined by $\left|\Phi_{A B}\right\rangle=\frac{1}{\sqrt{D}} \sum_{j=0}^{D-1}|j\rangle_{A} \otimes|j\rangle_{B}$, where $\left\{|j\rangle_{A}\right\}_{j=0}^{D-1}$ and $\left\{|j\rangle_{B}\right\}_{j=0}^{D-1}$ are respective orthonormal bases. Note that $I(A ; B)_{|\Phi\rangle\langle\Phi|}=2 \cdot \log (D)$ and $\left.I(A\rangle B\right)_{|\Phi\rangle\langle\Phi|}=\log (D)$.

The entanglement of formation of a joint state $\rho_{A B}$ is defined as ${ }^{98,107}$

$$
E_{F}\left(\rho_{A B}\right) \triangleq \inf _{p_{X}(x),\left|\psi_{A B}^{x}\right\rangle} H(A \mid X)_{\rho}
$$

where the infimum is over all pure state decompositions $\rho_{A B}=\sum_{x} p_{X}(x)\left|\psi_{A B}^{x}\right\rangle\left\langle\psi_{A B}^{x}\right|$.

\section{B. Quantum Broadcast Channel}

A quantum broadcast channel maps a quantum state at the sender system to a quantum state at the receiver systems. Here, we consider a channel with two receivers. Formally, a quantum broadcast channel is a linear, completely positive, trace-preserving map $\mathscr{N}_{A \rightarrow B_{1} B_{2}}$ corresponding to a quantum physical evolution. We assume that the channel is memoryless. That is, if the systems $A^{n}=\left(A_{1}, \ldots, A_{n}\right)$ are sent through $n$ channel uses, then the input state $\rho_{A^{n}}$ undergoes the tensor product mapping $\mathscr{N}_{A^{n} \rightarrow B_{1}^{n} B_{2}^{n}} \equiv \mathscr{N}_{A \rightarrow B_{1} B_{2}}^{\otimes n}$. The marginal channel $\mathscr{N}_{A \rightarrow B_{1}}^{(1)}$ is defined by

$$
\mathscr{N}_{A \rightarrow B_{1}}^{(1)}\left(\rho_{A}\right)=\operatorname{Tr}_{B_{2}}\left(\mathscr{N}_{A \rightarrow B_{1} B_{2}}\left(\rho_{A}\right)\right)
$$

for Receiver 1, and similarly $\mathscr{N}_{A \rightarrow B_{2}}^{(2)}$ for Receiver 2 . One may say that $\mathscr{N}_{A \rightarrow B_{1} B_{2}}$ is an extension of $\mathscr{N}_{A \rightarrow B_{1}}^{(1)}$ and $\mathscr{N}_{A \rightarrow B_{2}}^{(2)}$. We will consider a broadcast channel with conferencing where Receiver 1 can transmit classical information to Receiver 2 using a noiseless communication link of capacity $C_{12}$. We will denote this classical communication channel by $C C_{G \rightarrow G^{\prime}}$, where $G$ and $G^{\prime}$ represent the registers that store the conference message transmitted from Receiver 1 and received at Receiver 2, respectively. The transmitter, Receiver 1, and Reciever 2 are often called Alice, Bob 1, and Bob 2. 
A quantum broadcast channel has a Kraus representation,

$$
\mathscr{N}_{A \rightarrow B_{1} B_{2}}\left(\rho_{A}\right)=\sum_{j} N_{j} \rho_{A} N_{j}^{\dagger}
$$

for some set of operators $N_{j}$ such that $\sum_{j} N_{j}^{\dagger} N_{j}=\mathbb{1}$.

Remark 1. The classical broadcast channel is the special case where the input and the outputs can be represented by classical random variables $X$ and $Y_{1}, Y_{2}$, respectively, while the Kraus operators are $N_{x, y_{1}, y_{2}}=\sqrt{P_{Y_{1} Y_{2} \mid X}\left(y_{1}, y_{2} \mid x\right)}\left|y_{1}, y_{2}\right\rangle\langle x|$ for some probability kernel $P_{Y_{1} Y_{2} \mid X}$ and orthonormal bases $\{|x\rangle\},\left\{\left|y_{1}, y_{2}\right\rangle\right\}$. Therefore, given an input $x \in \mathscr{X}$, the output state of a classical broadcast channel is

$$
\mathscr{N}_{X \rightarrow Y_{1} Y_{2}}^{\mathrm{Cl}}(|x\rangle\langle x|)=\sum_{\left(y_{1}, y_{2}\right) \in \mathscr{Y}_{1} \times \mathscr{Y}_{2}} P_{Y_{1} Y_{2} \mid X}\left(y_{1}, y_{2} \mid x\right)\left|y_{1}, y_{2}\right\rangle\left\langle y_{1}, y_{2}\right| .
$$

\section{Degraded Broadcast Channel, Hadamard Broadcast Channel, and Degradable Marginals}

We will also be interested in the following special cases.

Definition 1 (Degraded broadcast channel and Hadamard broadcast channel ${ }^{58}$ ). A quantum broadcast channel $\mathscr{N}_{A \rightarrow B_{1} B_{2}}$ is called degraded if there exists a degrading channel $\mathscr{P}_{B_{1} \rightarrow B_{2}}$ such that the marginals satisfy the following relation,

$$
\mathscr{N}_{A \rightarrow B_{2}}^{(2)}=\mathscr{P}_{B_{1} \rightarrow B_{2}} \circ \mathscr{N}_{A \rightarrow B_{1}}^{(1)}
$$

In this case, we say that $\mathscr{N}^{(2)}$ is degraded with respect to $\mathscr{N}^{(1)}$. A quantum-classical-quantum degraded channel $\mathscr{N}_{A \rightarrow Y_{1} B_{2}}^{\mathrm{H}}$ is called a Hadamard broadcast channel.

Intuitively, if a broadcast channel is degraded, then the output state of Receiver 2 is a noisy version of that of Receiver 1. A Hadamard broadcast channel $\mathscr{N}_{A \rightarrow Y_{1} B_{2}}^{\mathrm{H}}$ can be viewed as a measureand-prepare channel where the marginal channel $\mathscr{N}_{A \rightarrow Y_{1}}^{(1)}$ acts as a measurement device, while the degrading channel $\mathscr{P}_{Y_{1} \rightarrow B_{2}}$ corresponds to state preparation. In this case, the marginal quantum channel $\mathscr{N}_{A \rightarrow B_{2}}^{(2)}$ of Receiver 2 is said to be entanglement-breaking ${ }^{108}$.

Next, we define a broadcast channel with degradable marginals. Every point-to-point quantum channel $\mathscr{M}_{A \rightarrow B}$ has an isometric extension $\mathscr{U}_{A \rightarrow B E}^{\mathscr{M}}\left(\rho_{A}\right)=U \rho_{A} U^{\dagger}$, also called a Stinespring dilation, where the operator $U$ is an isometry, i.e. $U^{\dagger} U=\mathbb{1}$ (see Ref. 109, Sec. VII). The system $E$ is 
often associated with the decoder's environment, or with a malicious eavesdropper in the wiretap channel model ${ }^{28}$. The channel $\widehat{\mathscr{M}}_{A \rightarrow E}\left(\rho_{A}\right)=\operatorname{Tr}_{B}\left(U \rho_{A} U^{\dagger}\right)$ is called the complementary channel for $\mathscr{M}_{A \rightarrow B}$.

Definition 2 (Degradable marginals). A point-to-point quantum channel $\mathscr{M}_{A \rightarrow B}$ is called degradable if there exists an isometric extension such that the complementary channel $\widehat{\mathscr{M}}_{A \rightarrow E}$ is degraded with respect to $\mathscr{M}_{A \rightarrow B}$. In other words, the channel to the environment is degraded with respect to the channel to the receiver. We say that the quantum broadcast channel $\mathscr{N}_{A \rightarrow B_{1} B_{2}}$ has degradable marginals if both marginals $\mathscr{N}_{A \rightarrow B_{1}}^{(1)}$ and $\mathscr{N}_{A \rightarrow B_{2}}^{(2)}$ are degradable.

Examples of degradable quantum channels include the erasure channel and the dephasing channel ${ }^{29}$.

\section{CODING FOR THE BROADCAST CHANNEL}

We consider different broadcast scenarios with cooperation between the decoders, where the transmitted information can be classical or quantum, with entanglement resources or without, and when conferencing between the receivers is available or not.

\section{A. Classical Coding with Entangled Decoders}

First, we consider a broadcast channel where Receiver 1 and Receiver 2 share entanglement resources. We denote their entangled systems by $S_{B_{1}}$ and $S_{B_{2}}$, respectively.

Definition 3. A $\left(2^{n R_{0}}, 2^{n R_{1}}, n\right)$ classical code for the quantum broadcast channel $\mathscr{N}_{A \rightarrow B_{1} B_{2}}$ with degraded message sets and entangled decoders consists of the following:

- Two index sets $\left[1: 2^{n R_{0}}\right]$ and $\left[1: 2^{n R_{1}}\right]$, corresponding to the common message for both users and the private message of User 1, respectively;

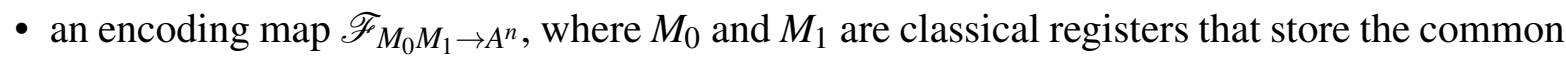
and private messages, respectively;

- a pure entangled state $\Psi_{S_{B_{1}}, S_{B_{2}}}$ 


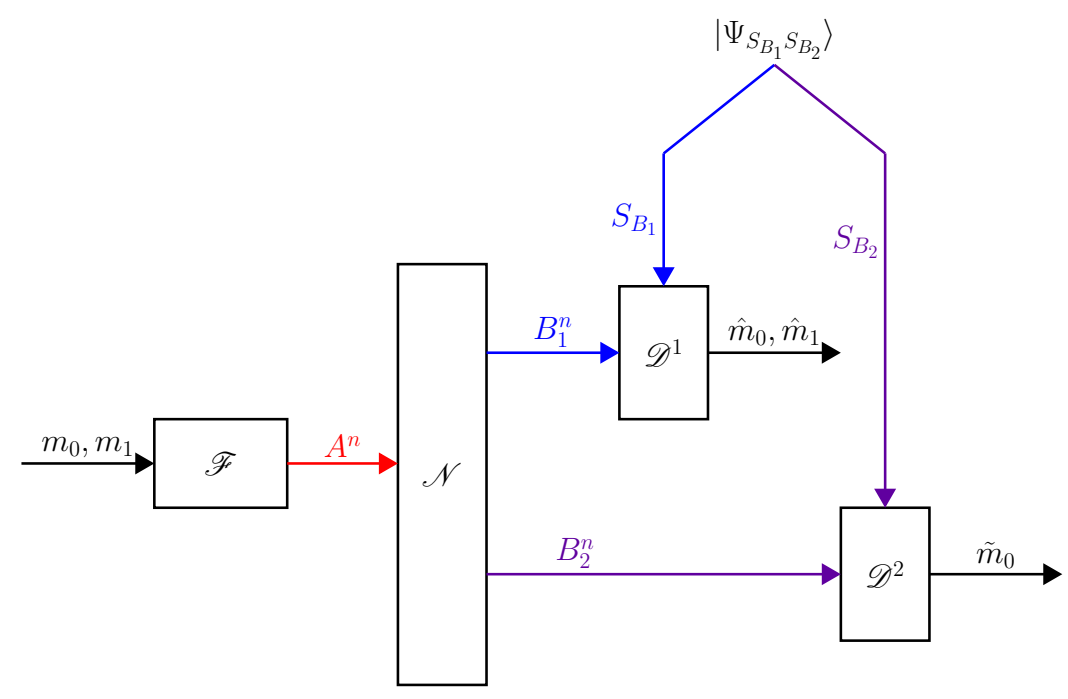

FIG. 1. Classical coding for a quantum broadcast channel $\mathscr{N}_{A \rightarrow B_{1} B_{2}}$ with shared entanglement between the decoders and degraded message sets. The quantum systems of Alice, Bob 1, and Bob 2 are marked in red, blue, and purple, respectively. Bob 1 and Bob 2 share entanglement resources in the systems $S_{B_{1}}$ and $S_{B_{2}}$, respectively. Alice encodes the messages $m_{0}$ and $m_{1}$ by applying the encoding map $\mathscr{F}_{M_{0} M_{1} \rightarrow A^{n}}$ to the respective registers $M_{0}$ and $M_{1}$ which store the messages. Then, she transmits the systems $A^{n}$ over the broadcast channel. The decoder $\mathscr{D}^{1}$ of Bob 1 receives the channel output systems $B_{1}^{n}$, and estimates the common and private messages by performing a decoding measurement on the systems $B_{1}^{n}$ and $S_{B_{1}}$, using a POVM $\left\{\Lambda_{S_{B_{1}} B_{1}^{n}}^{m_{1} m_{1}}\right\}$. Similarly, the decoder $\mathscr{D}^{2}$ of Bob 2 estimates the common message by measuring a $\operatorname{POVM}\left\{\Gamma_{S_{B_{2}} B_{2}^{n}}^{m_{0}}\right\}$ on $B_{2}^{n}$ and $S_{B_{2}}$.

- two decoding POVMs, $\left\{\Lambda_{S_{B_{1}} B_{1}^{n}}^{m_{0}, m_{1}}\right\}$ for Receiver 1 and $\left\{\Gamma_{S_{B_{2}} B_{2}^{n}}^{m_{0}}\right\}$ for Receiver 2, where the measurement outcome $m_{k}$ is an index in $\left[1: 2^{n R_{k}}\right]$, for $k=0,1$.

We denote the code by $(\mathscr{F}, \Psi, \Lambda, \Gamma)$.

The communication scheme is depicted in Figure 1. The sender Alice has the systems $A^{n}$, and the receivers Bob 1 and Bob 2 have the systems $B_{1}^{n}, S_{B_{1}}$ and $B_{2}^{n}, S_{B_{2}}$, respectively. Alice chooses a common message $m_{0} \in\left[1: 2^{n R_{0}}\right]$ that is intended for both users and a private message $m_{1} \in[1$ : $\left.2^{n R_{1}}\right]$ for Bob 1 , and stores them in the classical registers $M_{0}$ and $M_{1}$, respectively. She encodes the messages by applying the encoding map $\mathscr{F}_{M_{0} M_{1} \rightarrow A^{n}}$ which results in an input state $\rho_{A^{n}}^{m_{0}, m_{1}}=$ $\mathscr{F} M_{0} M_{1} \rightarrow A^{n}\left(m_{0}, m_{1}\right)$, and transmits the systems $A^{n}$ over $n$ channel uses of $\mathscr{N}_{A \rightarrow B_{1} B_{2}}$. Hence, the 
output state is

$$
\rho_{B_{1}^{n} B_{2}^{n}}^{m_{0}, m_{1}}=\mathscr{N}^{\otimes n}\left(\rho_{A^{n}}^{m_{0}, m_{1}}\right)
$$

Bob 1 receives the channel output systems $B_{1}^{n}$, combines them with his entangled system $S_{B_{1}}$, and applies the POVM $\left\{\Lambda_{S_{B_{1}} B_{1}^{n}}^{m_{0}, m_{1}}\right\}$. Bob 1 then obtains from the measurement outcome an estimate of the message pair $\left(\hat{m}_{0}, \hat{m}_{1}\right) \in\left[1: 2^{n R_{0}}\right] \times\left[1: 2^{n R_{1}}\right]$. Similarly, Bob 2 finds an estimate of the common message $\widetilde{m}_{0} \in\left[1: 2^{n R_{0}}\right]$ by performing a measurement using $\left\{\Gamma_{S_{B_{2}} B_{2}^{n}}^{m_{0}}\right\}$ on the output systems $B_{2}^{n}$ and his entangled system $S_{B_{2}}$. The conditional probability of error of the code, given that the message pair $\left(m_{0}, m_{1}\right)$ was sent, is given by

$$
P_{e \mid m_{0}, m_{1}}^{(n)}(\mathscr{F}, \Psi, \Lambda, \Gamma)=1-\operatorname{Tr}\left[\left(\Lambda_{S_{B_{1}} B_{1}^{n}}^{m_{0}, m_{1}} \otimes \Lambda_{S_{B_{1}} B_{2}^{n}}^{m_{0}}\right) \rho_{B_{1}^{n} B_{2}^{n}}^{m_{0}, m_{1}}\right]
$$

A $\left(2^{n R_{0}}, 2^{n R_{1}}, n, \varepsilon\right)$ classical code satisfies $P_{e \mid m_{0}, m_{1}}^{(n)}(\mathscr{F}, \Psi, \Lambda, \Gamma) \leq \varepsilon$ for all $\left(m_{0}, m_{1}\right) \in\left[1: 2^{n R_{0}}\right] \times$ $\left[1: 2^{n R_{1}}\right]$. A rate pair $\left(R_{0}, R_{1}\right)$ is called achievable with entangled decoders if for every $\varepsilon>0$ and sufficiently large $n$, there exists a $\left(2^{n R_{0}}, 2^{n R_{1}}, n, \varepsilon\right)$ code. The classical capacity region is defined as the set of achievable pairs $\left(R_{0}, R_{1}\right)$ with entangled decoders.

One may also consider the broadcast channel with independent messages, i.e. when the common message $m_{0}$ is replaced by a private message $m_{2}$ that is intended for Bob 2, in which case Bob 1 is not required to decode this message. In general, the capacity region with independent messages can be larger than with degraded message sets.

\section{B. Classical Coding with Conferencing}

Another form of cooperation between the decoders involves conferencing. We consider a broadcast channel where Receiver 1 can transmit information to Receiver 2 using a classical conferencing link of capacity $\mathrm{C}_{12}$.

Definition 4. A $\left(2^{n R_{0}}, 2^{n R_{1}}, n\right)$ classical code for the quantum broadcast channel $\mathscr{N}_{A \rightarrow B_{1} B_{2}}$ with degraded message sets and conferencing consists of the following:

- Three index sets $\left[1: 2^{n R_{0}}\right],\left[1: 2^{n R_{1}}\right]$, and $\left[1: 2^{n C_{12}}\right]$, corresponding to the common message for both users, the private message of User 1, and the conference message, respectively; 


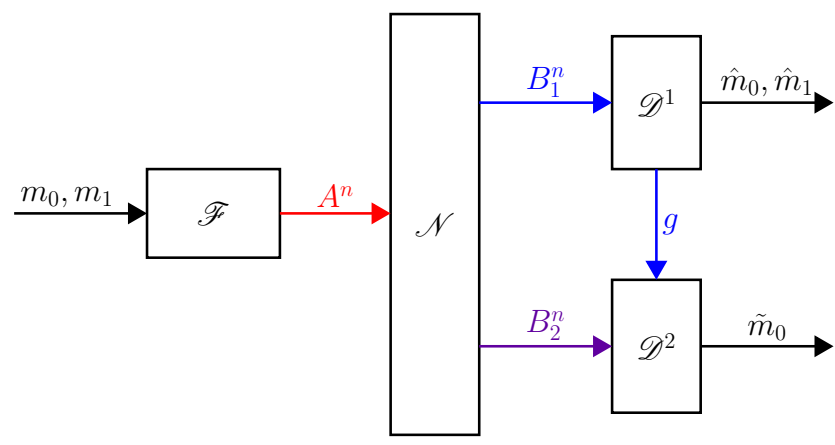

FIG. 2. Classical coding for a quantum broadcast channel $\mathscr{N}_{A \rightarrow B_{1} B_{2}}$ with conferencing and degraded message sets. The quantum systems of Alice, Bob 1, and Bob 2 are marked in red, blue, and purple, respectively. There is a conferencing link between the decoders which allows Bob 1 to send a conferencing message to Bob 2 at a rate $C_{12}$. Alice encodes the messages $m_{0}$ and $m_{1}$ by applying the encoding map $\mathscr{F}_{M_{0} M_{1} \rightarrow A^{n}}$ to the respective registers $M_{0}$ and $M_{1}$ which store the messages. Then, she transmits the systems $A^{n}$ over the broadcast channel. The decoder $\mathscr{D}^{1}$ of Bob 1 receives the channel output systems $B_{1}^{n}$ and performs a measurement using the POVM $\left\{\Lambda_{B_{1}^{n}}^{m_{0}, m_{1}, g}\right\}$. Bob 1 then obtains from the measurement outcome an estimate of the message pair and a conference message. Next, Bob 1 sends the conference message $g$ to Bob 2. Given the conference message $g$, the decoder $\mathscr{D}^{2}$ of Bob 2 chooses a measurement POVM $\left\{\Gamma_{B_{2}^{n} \mid g}^{m_{0}}\right\}$ to perform on the channel output systems $B_{2}^{n}$, producing an estimate of the common message as the measurement outcome.

- an encoding map $\mathscr{F}_{M_{0}} M_{1} \rightarrow A^{n}$, where $M_{0}$ and $M_{1}$ are classical registers that store the common and private messages, respectively;

- a decoding POVM $\left\{\Lambda_{B_{1}^{n}}^{m_{0}, m_{1}, g}\right\}$ for Receiver 1, where the measurement outcome is a triplet of indices $\left(m_{0}, m_{1}, g\right)$ in $\left[1: 2^{n R_{0}}\right] \times\left[1: 2^{n R_{1}}\right] \times\left[1: 2^{C_{12}}\right]$; and

- a collection of decoding POVMs $\left\{\Gamma_{B_{2}^{n} \mid g}^{m_{0}}\right\}, g \in\left[1: 2^{C_{12}}\right]$, for Receiver 2, where the measurement outcome is an index in $\left[1: 2^{n R_{0}}\right]$.

We denote the code by $(\mathscr{F}, \Lambda, \Gamma)$.

The communication scheme is depicted in Figure 2. The sender Alice has the systems $A^{n}$, and the receivers Bob 1 and Bob 2 have the systems $B_{1}^{n}$ and $B_{2}^{n}$, respectively. Alice chooses a common message $m_{0} \in\left[1: 2^{n R_{0}}\right]$ and a private message $m_{1} \in\left[1: 2^{n R_{1}}\right]$ for Bob 1 , and stores them in the classical registers $M_{0}$ and $M_{1}$, respectively. She encodes the messages by applying the encoding 
map $\mathscr{F}_{M_{0}} M_{1} \rightarrow A^{n}$ which results in an input state

$$
\rho_{A^{n}}^{m_{0}, m_{1}}=\mathscr{F} M_{0} M_{1} \rightarrow A^{n}\left(m_{0}, m_{1}\right)
$$

and transmits the systems $A^{n}$ over $n$ channel uses of $\mathscr{N}_{A \rightarrow B_{1} B_{2}}$. Hence, the output state is

$$
\rho_{B_{1}^{n} B_{2}^{n}}^{m_{0}, m_{1}}=\mathscr{N}_{A \rightarrow B_{1} B_{2}}^{\otimes n}\left(\rho_{A^{n}}^{m_{0}, m_{1}}\right)
$$

Bob 1 receives the channel output systems $B_{1}^{n}$ and applies the $\operatorname{POVM}\left\{\Lambda_{B_{1}^{n}}^{m_{0}, m_{1}, g}\right\}$. Bob 1 then obtains from the measurement outcome an estimate of the message pair $\left(\hat{m}_{0}, \hat{m}_{1}\right) \in\left[1: 2^{n R_{0}}\right] \times[1$ : $\left.2^{n R_{1}}\right]$ and a conference message $g \in\left[1: 2^{n \mathrm{C}_{12}}\right]$. Next, Bob 1 sends the conference message $g$ to Bob 2. Given the conference message $g$, Bob 2 chooses a POVM $\left\{\Gamma_{B_{2}^{n} \mid g}^{m_{0}}\right\}$ to perform on the channel output systems $B_{2}^{n}$, producing an estimate of the common message $\widetilde{m}_{0} \in\left[1: 2^{n R_{0}}\right]$ as the measurement outcome. The conditional probability of error of the code, given that the message pair $\left(m_{0}, m_{1}\right)$ was sent, is given by

$$
P_{e \mid m_{0}, m_{1}}^{(n)}(\mathscr{F}, \Lambda, \Gamma)=1-\sum_{g=1}^{2^{n C_{12}}} \operatorname{Tr}\left[\left(\Lambda_{B_{1}^{n}}^{m_{0}, m_{1}, g} \otimes \Lambda_{B_{2}^{n} \mid g}^{m_{0}}\right) \rho_{B_{1}^{n} B_{2}^{n}}^{m_{0}, m_{1}}\right]
$$

$\mathrm{A}\left(2^{n R_{0}}, 2^{n R_{1}}, n, \varepsilon\right)$ classical code satisfies $P_{e \mid m_{0}, m_{1}}^{(n)}(\mathscr{F}, \Lambda, \Gamma) \leq \varepsilon$ for all $\left(m_{0}, m_{1}\right) \in\left[1: 2^{n R_{0}}\right] \times[1$ : $2^{n R_{1}}$. A rate pair $\left(R_{0}, R_{1}\right)$ is called achievable with conferencing if for every $\varepsilon>0$ and sufficiently large $n$, there exists a $\left(2^{n R_{0}}, 2^{n R_{1}}, n, \varepsilon\right)$ code. The classical capacity region $\mathbb{R}_{\mathrm{Cl}}(\mathscr{N})$ is defined as the set of achievable pairs $\left(R_{0}, R_{1}\right)$ with conferencing.

Remark 2. The setting above is the quantum version of the classical broadcast channel with cooperating decoders, by Dabora and Servetto ${ }^{95}$. The main motivation involves a sensor network, where an external transmitter $\left(B_{1}\right)$ wants to download data such as network configuration into the network (see Ref. 95, Subsec. I A). The model can be viewed as a combination of the broadcast channel and the primitive relay channel ${ }^{97}$. In this context, the term 'conferencing' indicates cooperation between two different users, whereas a relay channel ${ }^{110}$ consists of a single user and a helper (see Def. 6).

Remark 3. The conferencing link can be described as a bit-pipe ${ }^{96}$, i.e. a noiseless link, from Receiver 1 to Receiver 2, through which information is transmitted at a constant rate $C_{12}$. 


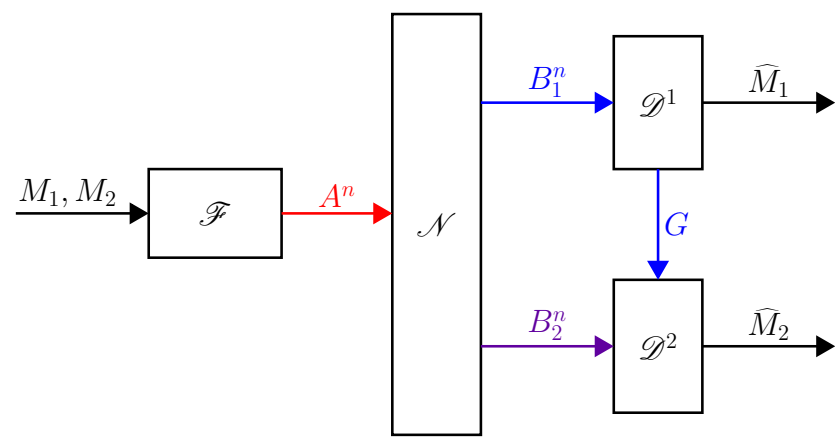

FIG. 3. Quantum coding for a broadcast channel $\mathscr{N}_{A \rightarrow B_{1} B_{2}}$ with conferencing and private messages. The quantum systems of Alice, Bob 1, and Bob 2 are marked in red, blue, and purple, respectively. Given entanglement between the decoders, the classical conference link can be used to transfer quantum information from Bob 1 to Bob 2 using the teleportation protocol. This is thus equivalent to a conferencing link with quantum capacity $C_{Q, 12}=\frac{1}{2} C_{12}$. Alice encodes the quantum state of the message systems $M_{1}$ and $M_{2}$ by

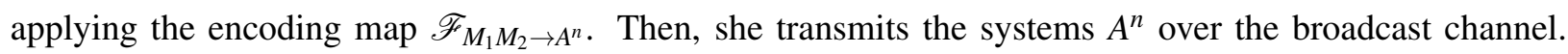
Bob 1 receives the channel output systems $B_{1}^{n}$ and applies the decoding map $\mathscr{D}_{B_{1}^{n} \rightarrow \widehat{M}_{1} G}^{1}$ such that the state of $\widehat{M}_{1}$ is his estimate of his private message. Next, Bob 1 sends the state of the conference system $G$ to Bob 2 through a noiseless conference link $\operatorname{id}_{G \rightarrow G^{\prime}}$. Bob 2 receives the channel output systems $B_{2}^{n}$ and the conference message in $G^{\prime}$, and applies the decoding map $\mathscr{D}_{G^{\prime} B_{2}^{n} \rightarrow \widehat{M}_{2}}^{2}$ such that the state of $\widehat{M}_{2}$ is his estimate of his private message.

\section{Quantum Coding with Conferencing, Entanglement Generation, and Entanglement Transmission}

Next, we consider the case where the messages are quantum. Furthermore, given entanglement between the decoders, the classical conference link can be used to transfer quantum information from Receiver 1 to Receiver 2 using the teleportation protocol. This is thus equivalent to a conferencing link with quantum capacity $C_{Q, 12}=\frac{1}{2} C_{12}$. In other words, given entanglement resources, the conferencing bit-pipe of capacity $\mathrm{C}_{12}$ can be transformed into a conferencing qubit-pipe of capacity $C_{Q, 12}=\frac{1}{2} C_{12}$ (see Rem. 3). Note that due to the no-cloning theorem, the encoder cannot transmit a quantum message to both receivers, thus we consider two private messages.

Definition 5. A $\left(2^{n Q_{1}}, 2^{n Q_{2}}, n\right)$ quantum code for the quantum broadcast channel $\mathscr{N}_{A \rightarrow B_{1} B_{2}}$ with independent messages and conferencing consists of the following: 
- A quantum message state $\rho_{M_{1} M_{2}}$, where $M_{1}$ and $M_{2}$ are quantum systems that store the private messages of User 1 and User 2, respectively. The dimension of each system is given by $\left|\mathscr{H}_{M_{k}}\right|=2^{n Q_{k}}$ for $k=1,2$.

- an encoding map $\mathscr{F}_{M_{1} M_{2} \rightarrow A^{n}}$;

- a decoding map $\mathscr{D}_{B_{1}^{n} \rightarrow \widehat{M}_{1} G}^{1}$ for Receiver 1 , where $G$ is a quantum register of dimension $2^{n C_{Q, 12}}$ that stores the conference message from Receiver 1 to Receiver 2;

- a decoding map $\mathscr{D}_{G^{\prime} B_{2}^{n} \rightarrow \widehat{M}_{2}}^{2}$ for Receiver 2 .

We denote the code by $\left(\mathscr{F}, \mathscr{D}^{1}, \mathscr{D}^{2}\right)$.

The communication scheme is depicted in Figure 3. The sender Alice has the systems $M_{1}$, $M_{2}$, and $A^{n}$; Bob 1 has the systems $B_{1}^{n}, G$, and $\widehat{M}_{1}$; and Bob 2 has the systems $B_{2}^{n}, G^{\prime}$, and $\widehat{M}_{2}$. Alice encodes the quantum state of the message systems $M_{1}$ and $M_{2}$ by applying the encoding map $\mathscr{F}_{M_{1}} M_{2} \rightarrow A^{n}$, which results in the input state

$$
\rho_{A^{n}}=\mathscr{F}_{M_{1} M_{2} \rightarrow A^{n}}\left(\rho_{M_{1} M_{2}}\right)
$$

and transmits the systems $A^{n}$ over $n$ channel uses of $\mathscr{N}_{A \rightarrow B_{1} B_{2}}$. Hence, the output state is

$$
\rho_{B_{1}^{n} B_{2}^{n}}=\mathscr{N}_{A \rightarrow B_{1} B_{2}}^{\otimes n}\left(\rho_{A^{n}}\right)
$$

Bob 1 receives the channel output systems $B_{1}^{n}$ and applies the decoding map $\mathscr{D}_{B_{1}^{n} \rightarrow \widehat{M}_{1} G}^{1}$, which results in

$$
\rho_{\widehat{M}_{1} G B_{2}^{n}}=\mathscr{D}_{B_{1}^{n} \rightarrow \widehat{M}_{1} G}^{1}\left(\rho_{B_{1}^{n} B_{2}^{n}}\right) .
$$

The reduced state of $\widehat{M}_{1}$ is Bob 1's estimate of the original state of his private message system $M_{1}$. Next, Bob 1 sends the conference message from $G$ to $G^{\prime}$ using the noiseless conference link $\operatorname{id}_{G \rightarrow G^{\prime}}$, hence $\rho_{\widehat{M}_{1} G^{\prime} B_{2}^{n}}=\operatorname{id}_{G \rightarrow G^{\prime}}\left(\rho_{\widehat{M}_{1} G B_{2}^{n}}\right)=\rho_{\widehat{M}_{1} G B_{2}^{n}}$. Bob 2 receives the channel output systems $B_{2}^{n}$ and the conference message in $G^{\prime}$, and applies the decoding map $\mathscr{D}_{G^{\prime} B_{2}^{n} \rightarrow \widehat{M}_{2}}^{2}$ such that $\widehat{M}_{2}$ is his estimate of his private message. The estimated state is then given by

$$
\rho_{\widehat{M}_{1} \widehat{M}_{2}}=\mathscr{D}_{G^{\prime} B_{2}^{n} \rightarrow \widehat{M}_{2}}^{2}\left(\rho_{\widehat{M}_{1} G B_{2}^{n}}\right)
$$

and the estimation error by

$$
e^{(n)}\left(\mathscr{E}, \mathscr{D}^{1}, \mathscr{D}^{2}, \rho_{M_{1}, M_{2}}\right)=\frac{1}{2}\left\|\rho_{M_{1} M_{2}}-\rho_{\widehat{M}_{1} \widehat{M}_{2}}\right\|
$$


A $\left(2^{n Q_{1}}, 2^{n Q_{2}}, n, \varepsilon\right)$ quantum code satisfies $e^{(n)}\left(\mathscr{F}, \mathscr{D}^{1}, \mathscr{D}^{2}, \rho_{M_{0} M_{1}}\right) \leq \varepsilon$ for all $\rho_{M_{0}, M_{1}}$. A rate pair $\left(Q_{1}, Q_{2}\right)$ is called achievable with conferencing if for every $\varepsilon>0$ and sufficiently large $n$, there exists a $\left(2^{n Q_{1}}, 2^{n Q_{2}}, n, \varepsilon\right)$ code. The quantum capacity region $\mathbb{R}_{\mathrm{Q}}(\mathscr{N})$ is defined as the set of achievable pairs $\left(Q_{1}, Q_{2}\right)$ with conferencing.

The setting of a broadcast channel with conferencing is closely related to that of a primitive relay channel ${ }^{97}$.

Definition 6. A primitive relay channel $\mathscr{N}_{A \rightarrow B_{1} B_{2}}^{\text {relay }}$ is a broadcast channel with conferencing, when User 1 does not send information, i.e. $Q_{1}=0$. Alice, Bob 1 , and Bob 2 are then called the source, relay, and destination receiver, respectively. A quantum rate $Q_{2}>0$ is called achievable for the primitive relay channel if $\left(0, Q_{2}\right)$ is achievable for the broadcast channel with conferencing. The quantum capacity $C_{\mathrm{Q}}\left(\mathscr{N}^{\text {relay }}\right)$ is defined as the supremum of achievable rates for the primitive relay channel.

Bob 1 is called a relay in this setting, because his only task is to help the transmission of information to Bob 2 (see Figure 4). The channel is called 'primitive' since it is a simplified version of the (non-primitive) relay channel ${ }^{110}$ where information is received and encoded at the relay in a strictly-causal manner.

Remark 4. A standard, i.e. non-primitive, relay channel ${ }^{89-91}$ is specified by a linear, completely positive, trace-preserving map $\mathscr{L}_{A \rightarrow B_{1} A_{1} B_{2}}$, where the sender transmits the systems $A^{n}$, the relay receives $B_{1}^{n}$ and transmits $A_{1}^{n}$, and the destination receiver receives $B_{2}^{n}$. The relay encoder applies a strictly-causal map, as he can only use the systems $B_{1}^{i-1}$ at time $i$. That is, at time $i$, the relay transmits $A_{1, i}$ such that $\rho_{A_{1}^{i}}=\mathscr{T}_{B_{1}^{i-1} \rightarrow A_{1}^{i}}^{(i)}\left(\rho_{B_{1}^{i-1}}\right)$.

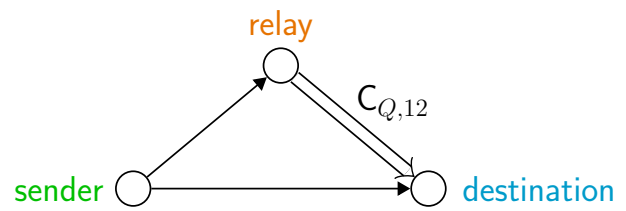

FIG. 4. A simplistic view of the primitive relay channel. Alice, Bob 1, and Bob 2 play the roles of the sender, relay, and destination receiver, respectively. 
Remark 5. Quantum communication is also referred to as entanglement transmission and can be extended to strong subspace transmission ${ }^{31,111}$. In this task, Alice and Charlie share a pure entangled state $\left|\psi_{M_{1} M_{2} C}\right\rangle$, with a Schmidt decoposition

$$
\left|\psi_{M_{1} M_{2} C}\right\rangle=\sum_{x \in \mathscr{X}} \sqrt{p_{X}(x)}\left|\psi_{M_{1} M_{2}}^{x}\right\rangle \otimes\left|\psi_{C}^{x}\right\rangle
$$

Given a $\left(2^{n Q_{1}}, 2^{n Q_{2}}, n, \varepsilon\right)$ code, Alice can send each state $\left|\psi_{M_{1} M_{2}}^{x}\right\rangle$ reliably, i.e. with

$$
\left\|\psi_{M_{1} M_{2}}^{x}-\psi_{\widehat{M}_{1} \widehat{M}_{2}}^{x}\right\|_{1} \leq \varepsilon \text { for all } x \in \mathscr{X}
$$

Hence, the superposition state $\left|\psi_{M_{1} M_{2} C}\right\rangle$ can also be recovered up to an error of $\varepsilon$. In particular, if Alice and Charlie share a maximally entangled state $\left|\Phi_{M_{1} M_{2} C}\right\rangle$, then at the end of the communication protocol, Bob 1, Bob 2, and Charlie share a state $\approx\left|\Phi_{\widehat{M}_{1} \widehat{M}_{2} C}\right\rangle$ up to an $\varepsilon$-error.

Remark 6. Quantum communication can also be used for the purpose of entanglement generation ${ }^{28,31}$. We note that by the monogamy property of quantum entanglement ${ }^{79}$, Alice cannot generate a maximally entangled state with both Bob 1 and Bob 2 simultaneously. Indeed, suppose that Alice has a third system $\bar{A}$ that is entangled with $M_{1}$ and $M_{2}$ in a state $\left|\psi_{\bar{A} M_{1} M_{2}}\right\rangle$. Then, by strong sub-additivity (Ref. 36, Coro. 11.9.1),

$$
H\left(\bar{A} M_{1}\right)_{\psi}+H\left(\bar{A} M_{1}\right)_{\psi} \geq H(\bar{A})_{\psi}+H\left(\bar{A} M_{1} M_{2}\right)_{\psi} \geq H(\bar{A})_{\psi}
$$

Hence, $\bar{A}$ cannot be maximally entangled with both $M_{1}$ and $M_{2}$, otherwise we would have $0 \geq 1$. Nevertheless, different forms of entanglement can be generated. In particular, Alice can generate a GHZ state with Bob 1 and Bob 2 (Ref. 55, Sec. IV), using $\left|\psi_{\bar{A} M_{1} M_{2}}\right\rangle=\frac{1}{\sqrt{d}} \sum_{x=1}^{d}|x\rangle \otimes|x\rangle \otimes|x\rangle$. Alternatively, she can generate two entangled pairs. Suppose that Alice has another pair of system $\bar{A}_{1}, \bar{A}_{2}$ in the state

$$
\left|\psi_{\bar{A}_{1} M_{1} \bar{A}_{2} M_{2}}\right\rangle=\left|\Phi_{\bar{A}_{1} M_{1}}\right\rangle \otimes\left|\Phi_{\bar{A}_{2} M_{2}}\right\rangle .
$$

Then, at the end of the quantum communication protocol, Alice shares the entangled states $\approx$ $\left|\Phi_{\bar{A}_{1} \widehat{M}_{1}}\right\rangle$ with Bob 1 and $\approx\left|\Phi_{\bar{A}_{2} \widehat{M}_{2}}\right\rangle$ with Bob 2, by the same considerations as in entanglement transmission (see Remark 5).

Remark 7. In the absence of entanglement resources between the decoders, quantum communication over the broadcast channel can generate such entanglement by choosing the quantum message state to be $\left|\Phi_{M_{1} M_{2}}\right\rangle$. 


\section{MAIN RESULTS - CLASSICAL CONFERENCING}

Now, we give our results on the quantum broadcast channel with a classical conferencing link between the decoders, when Bob 1 and Bob 2 do not share entanglement resources (see Fig. 2). Define the rate region

$$
\mathscr{R}_{\mathrm{Cl}}(\mathscr{N}) \triangleq \bigcup\left\{\begin{array}{c}
\left(R_{0}, R_{1}\right): \quad \\
R_{0} \leq I\left(X_{0} ; B_{2}\right)_{\rho}+\mathrm{C}_{12} \\
R_{1} \leq I\left(X_{1} ; B_{1} \mid X_{0}\right) \\
R_{0}+R_{1} \leq I\left(X_{0}, X_{1} ; B_{1}\right)
\end{array}\right\}
$$

where the union is over the set of all distributions $p_{X_{0}, X_{1}}\left(x_{0}, x_{1}\right)$ and state collection $\left\{\theta_{A}^{x_{0}, x_{1}}\right\}$, with

$$
\rho_{X_{0} X_{1} B}=\sum_{x_{0} \in \mathscr{X}_{0}} \sum_{x_{1} \in \mathscr{X}_{1}} p_{X_{0}, X_{1}}\left(x_{0}, x_{1}\right)\left|x_{0}\right\rangle\left\langle x_{0}|\otimes| x_{1}\right\rangle\left\langle x_{1}\right| \otimes \mathscr{N}_{A \rightarrow B_{1} B_{2}}\left(\theta_{A}^{x_{0}, x_{1}}\right) .
$$

Before we state the capacity theorem, we give the following lemma which provides cardinality bounds for the auxiliary random variables $X_{0}$ and $X_{1}$. In principle, one can use those cardinality bounds to evaluate the region $\mathscr{R}_{\mathrm{Cl}}(\mathscr{N})$ numerically.

Lemma 1. The union in (23) is exhausted by auxiliary random variables $X_{0}$ and $X_{1}$ of cardinality $\left|\mathscr{X}_{0}\right| \leq\left|\mathscr{H}_{A}\right|^{2}+2$ and $\left|\mathscr{X}_{1}\right| \leq\left(\left|\mathscr{H}_{A}\right|^{2}+2\right)\left|\mathscr{H}_{A}\right|^{2}+1$.

The proof of Lemma 1 is given in Appendix A. The classical capacity region is determined in the theorem below.

Theorem 2. The classical capacity region of the quantum broadcast channel $\mathscr{N}_{A \rightarrow B_{1} B_{2}}$ with conferencing and degraded message sets is given by

$$
\mathbb{R}_{\mathrm{Cl}}(\mathscr{N})=\lim _{k \rightarrow \infty} \bigcup_{k=1}^{\infty} \mathscr{R}_{\mathrm{Cl}}\left(\mathscr{N}^{\otimes k}\right) .
$$

Furthermore, for a Hadamard broadcast channel,

$$
\mathbb{R}_{\mathrm{Cl}}(\mathscr{N})=\mathscr{R}_{\mathrm{Cl}}(\mathscr{N})
$$

The proof of Theorem 2 is given in Appendix B.

Note that in the special case of a conference link with zero capacity, i.e. $C_{12}=0$, we recover the result by Yard et al. ${ }^{75}$ on the broadcast channel without conferencing. 


\section{MAIN RESULTS - QUANTUM CONFERENCING}

Next, we consider the case where the messages are quantum. Furthermore, given entanglement between the decoders, the classical conference link can be used to transfer quantum information from Receiver 1 to Receiver 2 using the teleportation protocol. This is thus equivalent to a conferencing link with quantum capacity $C_{Q, 12}=\frac{1}{2} C_{12}$. As noted in Subsection III C, Alice cannot transmit a quantum message to both receivers due to the no-cloning theorem. Thereby, we consider a broadcast channel with two private quantum messages, as illustrated in Fig. 3.

This setting is intimately related to quantum repeaters, as Bob 1 can be viewed as a repeater for the transmission of quantum information to Bob 2. In particular, Alice can use the quantum message stored in $M_{1}$ to generate entanglement and prepare a maximally entangled pair $\left|\Phi_{A B_{1}}\right\rangle$ between the transmitter and the repeater, namely, Alice and Bob 1. Given entanglement between the decoders, we also have a maximally entangled pair $\left|\Phi_{B_{1}^{\prime} B_{2}^{\prime}}\right\rangle$ between the repeater and the receiver, i.e. Bob 1 and Bob 2. Then, the repeater $B_{1}$ can swap his entanglement by using the classical conferencing link to teleport the state of $B_{1}^{\prime}$ onto $B_{2}$ thus swapping the entanglement such that $A$ and $B_{2}$ are now entangled. This requires that the conferencing capacity is at least twice the information rate, i.e. $C_{12} \geq 2 Q_{2}$. We will conclude this section with the resulting observations for the quantum repeater.

\section{A. Achievable Region}

We establish an achievable rate region for the broadcast channel with quantum conferencing.

Theorem 3. A rate pair $\left(Q_{1}, Q_{2}\right)$ is achievable for transmission of quantum information over the broadcast channel $\mathscr{N}_{A \rightarrow B_{1} B_{2}}$ with private messages and quantum conferencing if

$$
\begin{aligned}
Q_{1} & \left.\leq I\left(A_{1}\right\rangle B_{1}\right) \rho \\
Q_{2} & \left.\leq I\left(A_{2}\right\rangle B_{2}\right) \rho+C_{Q, 12} \\
Q_{1}+Q_{2} & \left.\left.\leq I\left(A_{1}\right\rangle B_{1}\right)_{\rho}+I\left(A_{2}\right\rangle B_{2}\right) \rho
\end{aligned}
$$

for some input state $\rho_{A_{1} A_{2} A^{\prime}}$, where $\rho_{A_{1} A_{2} B_{1} B_{2}}=\mathscr{N}_{A^{\prime} \rightarrow B_{1} B_{2}}\left(\rho_{A_{1} A_{2} A^{\prime}}\right)$.

The achievability proof is given below. The rate region in Theorem 3 reflects a greedy approach, where using the conferencing link to increase the information rate of User 2 comes directly at the expense of User 1. That is, if $\left.Q_{2}=I\left(A_{2}\right\rangle B_{2}\right)_{\rho}+\Delta$, then $\left.Q_{1} \leq I\left(A_{1}\right\rangle B_{1}\right)_{\rho}-\Delta$. 
Remark 8. For the transmission of classical information, we have seen that the optimal performance is achieved using superposition coding, where Receiver 1 can recover the message of User 2 without necessarily "losing" rate. In particular, by Theorem 2, a classical rate pair $\left(R_{1}, R_{2}\right)=$ $\left(I\left(X_{1} ; B_{1} \mid X_{0}\right)_{\rho}, I\left(X_{0} ; B_{2}\right)_{\rho}+C_{12}\right)$ is achievable when $I\left(X_{0} ; B_{2}\right)_{\rho}+C_{12}<I\left(X_{0} ; B_{1}\right)_{\rho}$, because then $R_{1}+R_{2}<I\left(X_{0} X_{1} ; B_{1}\right)$ by the chain rule. However, in the quantum case, the capacity-achieving coding scheme in Ref. 59 does not involve superposition. Without conferencing, it is impossible for Receiver 1 to decode the message of User 2 by the no-cloning theorem. Nevertheless, the setting of conferencing decoders imposes a chronological order: First Bob 1 receives and processes the channel output $B_{1}^{n}$, then Bob 1 sends the conference message to Bob 2, and at last, Bob 2 receives access to the channel output $B_{2}^{n}$ and the conference message. Therefore, Bob 1 can recover the state of $M_{2}$ (or part of it) and send it to Bob 2 using the conference link. However, due to the no-cloning theorem, Bob 2 will be able to decode the state of $M_{2}$ only if the state was destroyed in Bob 1's location during conferencing.

Achievability Proof. Consider the quantum broadcast channel $\mathscr{N}_{A \rightarrow B_{1} B_{2}}$ with a quantum conference link of capacity $C_{Q, 12}$. The proof is a straightforward consequence of the results by Dupuis $e t$ $a l .{ }^{59}$. Fix an input state $\rho_{A_{1} A_{2} A^{\prime}}$. Based on ${ }^{59}$ (Ref. 60, Theo. 5.4), for every $\varepsilon>0$ and sufficiently large $n$ there exists a $\left(2^{n Q_{1}^{\prime}}, 2^{n Q_{2}^{\prime}}, n, \varepsilon\right)$ quantum code for the broadcast channel $\mathscr{N}_{A \rightarrow B_{1} B_{2}}$ without conferencing if

$$
\begin{aligned}
& \left.Q_{1}^{\prime}=I\left(A_{1}\right\rangle B_{1}\right)_{\rho}-\delta \\
& \left.Q_{2}^{\prime}=I\left(A_{2}\right\rangle B_{2}\right)_{\rho}-\delta
\end{aligned}
$$

where $\delta>0$ is arbitrarily small. The rate pair $\left(Q_{1}^{\prime}, Q_{2}^{\prime}\right)$ is thus achievable in our setting as well, since the decoders can avoid conferencing by choosing an idle conference message state $|0\rangle\langle 0|$ regardless of the output state.

Now, we consider two cases: $\left.\mathrm{C}_{Q, 12}>I\left(A_{1}\right\rangle B_{1}\right)_{\rho}$ and $\left.\mathrm{C}_{Q, 12} \leq I\left(A_{1}\right\rangle B_{1}\right)_{\rho}$. If $\left.\mathrm{C}_{Q, 12}>I\left(A_{1}\right\rangle B_{1}\right)_{\rho}$, then the second inequality in (27) is inactive since $\left.\left.\left.I\left(A_{1}\right\rangle B_{1}\right)_{\rho}+I\left(A_{2}\right\rangle B_{2}\right) \rho<I\left(A_{2}\right\rangle B_{2}\right)_{\rho}+C_{Q, 12}$, hence we are done. Otherwise, if $\left.C_{Q, 12} \leq I\left(A_{1}\right\rangle B_{1}\right)_{\rho}$, then Alice can send the state of $n C_{Q, 12}$ qubits to Bob 2 indirectly through the conference link. This can be performed as follows. First, use the code above to transmit $n\left(Q_{1}^{\prime \prime}+\mathrm{C}_{Q, 12}\right)$ qubits to Bob 1 and $n Q_{2}^{\prime}$ to Bob 2, with $Q_{1}^{\prime \prime}=Q_{1}^{\prime}-\mathrm{C}_{Q, 12}$, and then let Bob 1 send the state of the $n \mathrm{C}_{Q, 12}$ qubits to Bob 2. Overall, this coding scheme achieves 
the following rate pair,

$$
\begin{aligned}
& \left.Q_{1}^{\prime \prime}=I\left(A_{1}\right\rangle B_{1}\right)_{\rho}-C_{Q, 12}-\delta \\
& \left.Q_{2}^{\prime \prime}=I\left(A_{2}\right\rangle B_{2}\right)_{\rho}+C_{Q, 12}-\delta
\end{aligned}
$$

Note that in the process of sending the conference message, Bob 1 may destroy the state of his own $n \mathrm{C}_{Q, 12}$ qubits, and thus this cannot be regarded as a common message. Observing that $\left(Q_{1}^{\prime}, Q_{2}^{\prime}\right)$ and $\left(Q_{1}^{\prime \prime}, Q_{2}^{\prime \prime}\right)$ are the corner points of the region in (27), the proof follows by time sharing.

Note that for $C_{12}=0$, the achievable region coincides with the capacity region of the broadcast channel without conferencing 59,60 .

\section{B. Outer Bound}

Next, we give a multi-letter outer bound.

Theorem 4. If a rate pair $\left(Q_{1}, Q_{2}\right)$ is achievable for transmission of quantum information over the broadcast channel $\mathscr{N}_{A \rightarrow B_{1} B_{2}}$ with private messages and quantum conferencing, then it must satisfy the following inequalities,

$$
\begin{aligned}
Q_{1} & \left.\leq \frac{1}{n} I\left(A_{1}\right\rangle B_{1}^{n}\right)_{\rho} \\
Q_{2} & \left.\leq \frac{1}{n} I\left(A_{2} T\right\rangle B_{2}^{n}\right)_{\rho}+\mathrm{C}_{Q, 12} \\
Q_{1}+Q_{2} & \left.\left.\leq \frac{1}{n} I\left(A_{1}\right\rangle B_{1}^{n}\right)_{\rho}+\frac{1}{n} I\left(A_{2}\right\rangle B_{1}^{n} B_{2}^{n}\right)_{\rho}
\end{aligned}
$$

for some input state $\rho_{T A_{1} A_{2} A^{\prime n}}$, where $\rho_{T A_{1} A_{2} B_{1}^{n} B_{2}^{n}}=\mathscr{N}_{A^{\prime} \rightarrow B_{1} B_{2}}^{\otimes n}\left(\rho_{T A_{1} A_{2} A^{\prime n}}\right)$.

Notice that here we added the auxiliary system $T$ in the second inequality and added $B_{1}^{n}$ in the last term of the third inequality (cf. (27) and (30)).

Proof of Outer Bound. Suppose that Alice is trying to generate entanglement with Bob 1 and Bob 2. An upper bound on the rate at which Alice and Bob $k$, for $k=1,2$, can generate entanglement also serves as an upper bound on the rate at which they can communicate qubits, since a noiseless quantum channel can be used to generate entanglement by sending one part of an entangled pair. In this task, Alice locally prepares two maximally entangled pairs,

$$
\left|\Phi_{M_{1} M_{1}^{\prime}}\right\rangle \otimes\left|\Phi_{M_{2} M_{2}^{\prime}}\right\rangle=\frac{1}{\sqrt{2^{n\left(Q_{1}+Q_{2}\right)}}} \sum_{m_{1}=1}^{2^{n Q_{1}}} \sum_{m_{2}=1}^{2^{n Q_{2}}}\left|m_{1}\right\rangle_{M_{1}} \otimes\left|m_{1}\right\rangle_{M_{1}^{\prime}} \otimes\left|m_{2}\right\rangle_{M_{2}} \otimes\left|m_{2}\right\rangle_{M_{2}^{\prime}}
$$


Quantum Broadcast Channels with Cooperating Decoders

Then, she applies an encoding channel $\mathscr{F} M_{1}^{\prime} M_{2}^{\prime} \rightarrow A^{\prime n}$ to the quantum systems $M_{1}^{\prime} M_{2}^{\prime}$, resulting in

$$
\rho_{M_{1} M_{2} A^{\prime n}} \equiv \mathscr{F}_{M_{1}^{\prime} M_{2}^{\prime} \rightarrow A^{\prime n}}\left(\left|\Phi_{M_{1} M_{1}^{\prime}}\right\rangle \otimes\left|\Phi_{M_{2} M_{2}^{\prime}}\right\rangle\right)
$$

After Alice sends the systems $A^{\prime n}$ through the channel, Bob 1 receives the systems $B_{1}^{n}$ in the state

$$
\rho_{M_{1} M_{2} B_{1}^{n} B_{2}^{n}} \equiv \mathscr{N}_{A^{\prime} \rightarrow B_{1} B_{2}}^{\otimes n}\left(\rho_{M_{1} M_{2} A^{\prime n}}\right)
$$

and performs a decoding channel $\mathscr{D}_{B_{1}^{n} \rightarrow \widehat{M}_{1} G}$. Hence,

$$
\rho_{M_{1} M_{2} \widehat{M}_{1} G B_{2}^{n}} \equiv \mathscr{D}_{B_{1}^{n} \rightarrow \widehat{M}_{1} G}\left(\rho_{M_{1} M_{2} B_{1}^{n} B_{2}^{n}}\right)
$$

where the state of $\widehat{M}_{1}$ is Bob 1's estimate of his quantum message, and the state of $G$ is the conference message which is sent through the conference link to Bob 2. Having received $B_{2}^{n}$ and $G^{\prime}$ such that $\rho_{M_{1} M_{2} \widehat{M}_{1} G^{\prime}}=\rho_{M_{1} M_{2} \widehat{M}_{1} G}$, Bob 2 uses a decoding channel $\mathscr{D}_{G^{\prime} B_{2}^{n} \rightarrow \widehat{M}_{2}}^{2}$, producing

$$
\rho_{M_{1} M_{2} \widehat{M}_{1} \widehat{M}_{2}} \equiv \mathscr{D}_{G^{\prime} B_{2}^{n} \rightarrow \widehat{M}_{2}}^{2}\left(\rho_{M_{1} M_{2} \widehat{M}_{1} G B_{2}^{n}}\right)
$$

Consider a sequence of codes $\left(\mathscr{F}_{n}, \mathscr{D}_{n}^{1}, \mathscr{D}_{n}^{2}\right)$ for entanglement generation, such that

$$
\frac{1}{2}\left\|\rho_{M_{1} \widehat{M}_{1} M_{2} \widehat{M}_{2}}-\Phi_{M_{1} M_{1}^{\prime}} \otimes \Phi_{M_{2} M_{2}^{\prime}}\right\|_{1} \leq \alpha_{n}
$$

where $\alpha_{n}$ tends to zero as $n \rightarrow \infty$. By the Alicki-Fannes-Winter inequality ${ }^{12,113}$ (Ref. 36, Theo. 11.10.3), (36) implies that $\left|H\left(M_{k} \mid \widehat{M}_{k}\right)_{\rho}-H\left(M_{k} \mid M_{k}^{\prime}\right)_{\Phi}\right| \leq n \varepsilon_{n}$, or equivalently,

$$
\left.\left.\mid I\left(M_{k}\right\rangle \widehat{M}_{k}\right)_{\rho}-I\left(M_{k}\right\rangle M_{k}^{\prime}\right)_{\Phi} \mid \leq n \varepsilon_{n}
$$

for $k=1,2$, where $\varepsilon_{n}$ tends to zero as $n \rightarrow \infty$. Observe that $\left.I\left(M_{k}\right\rangle M_{k}^{\prime}\right)_{\Phi}=H\left(M_{k}\right)_{\Phi}-H\left(M_{k} M_{k}^{\prime}\right)_{\Phi}=$ $n Q_{k}-0=n Q_{k}$. Thus,

$$
\begin{aligned}
n Q_{1} & \left.=I\left(M_{1}\right\rangle M_{1}^{\prime}\right)_{\Phi} \\
& \left.\leq I\left(M_{1}\right\rangle \widehat{M}_{1}\right)_{\rho}+n \varepsilon_{n} \\
& \left.\leq I\left(M_{1}\right\rangle B_{1}^{n}\right)_{\rho}+n \varepsilon_{n}
\end{aligned}
$$

where the last inequality is due to (34) and the data processing inequality for the coherent information (Ref. 36, Theo. 11.9.3). 
Quantum Broadcast Channels with Cooperating Decoders

Similarly, for User 2, it follows from (35) and the data processing inequality that

$$
\begin{aligned}
n Q_{2} & \left.\leq I\left(M_{2}\right\rangle G^{\prime} B_{2}^{n}\right) \rho+n \varepsilon_{n} \\
& =-H\left(M_{2} G^{\prime} \mid B_{2}^{n}\right)+H\left(G^{\prime} \mid B_{2}^{n}\right)+n \varepsilon_{n} \\
& \left.=I\left(M_{2} G^{\prime}\right\rangle B_{2}^{n}\right)+H\left(G^{\prime} \mid B_{2}^{n}\right)+n \varepsilon_{n} \\
& \left.\leq I\left(M_{2} G^{\prime}\right\rangle B_{2}^{n}\right)+n C_{Q, 12}+n \varepsilon_{n}
\end{aligned}
$$

By (38) and (39), we also have that

$$
\begin{aligned}
n\left(Q_{1}+Q_{2}\right) & \left.\left.\leq I\left(M_{1}\right\rangle B_{1}^{n}\right)_{\rho}+I\left(M_{2}\right\rangle G^{\prime} B_{2}^{n}\right)_{\rho}+2 n \varepsilon_{n} \\
& \left.\left.\leq I\left(M_{1}\right\rangle B_{1}^{n}\right)_{\rho}+I\left(M_{2}\right\rangle B_{1}^{n} B_{2}^{n}\right)_{\rho}+2 n \varepsilon_{n}
\end{aligned}
$$

where the last follows from (34) and the data processing inequality. The proof follows from (38), (40) and (41) by defining quantum systems $A_{1}, A_{2}$ such that for some isometries $U_{M_{1} \rightarrow A_{1}}, V_{M_{2} \rightarrow A_{2}}$ and $W_{G^{\prime} \rightarrow T}$, we have $\rho_{A_{1} B_{1}^{n}}=U_{M_{1} \rightarrow A_{1}^{n}} \rho_{M_{1} B_{1}^{n}} U_{M_{1} \rightarrow A_{1}^{n}}^{\dagger}$ and $\rho_{A_{2} T B_{2}^{n}}=\left(V_{M_{2} \rightarrow A_{2}} \otimes W_{G^{\prime} \rightarrow T}\right) \rho_{M_{2} G^{\prime} B_{2}^{n}}$ $\left(V_{M_{2} \rightarrow A_{2}} \otimes W_{G^{\prime} \rightarrow T}\right)^{\dagger}$. This completes the proof for the regularized outer bound.

\section{Primitive Relay Channel}

Consider the primitive relay channel $\mathscr{N}_{A \rightarrow B_{1} B_{2}}^{\text {relay }}$, where Bob 1 acts as a relay that helps the transmission from Alice to Bob 2, but is not required to decode information (i.e. $Q_{1}=0$ ). We use our previous results to obtain lower and upper bounds on the capacity of the primitive relay channel, and conclude this section with the resulting observations for the quantum repeater.

Theorem 5. The quantum capacity of the primitive relay channel $\mathscr{N}_{A \rightarrow B_{1} B_{2}}^{\text {relay }}$ has the following bounds:

1) Cutset upper bound

$$
\left.\left.C_{\mathrm{Q}}\left(\mathscr{N}^{\text {relay }}\right) \leq \lim _{n \rightarrow \infty} \sup _{\rho_{A T A^{\prime n}}} \frac{1}{n} \min \left[I(A T\rangle B_{2}^{n}\right)_{\rho}+C_{Q, 12}, I(A\rangle B_{1}^{n} B_{2}^{n}\right)_{\rho}\right]
$$

with $\rho_{A T B_{1}^{n} B_{2}^{n}}=\mathscr{N}_{A^{\prime} \rightarrow B_{1} B_{2}}^{\otimes n}\left(\rho_{A T A^{\prime n}}\right)$.

2) Decode-forward lower bound

$$
\left.\left.C_{\mathrm{Q}}\left(\mathscr{N}^{\text {relay }}\right) \geq \max _{\left|\phi_{A_{1} A_{2} A^{\prime}}\right\rangle}\left[I\left(A_{2}\right\rangle B_{2}\right)_{\rho}+\min \left(I\left(A_{1}\right\rangle B_{1}\right) \rho, C_{Q, 12}\right)\right]
$$

with $\rho_{A_{1} A_{2} B_{1} B_{2}}=\mathscr{N}_{A^{\prime} \rightarrow B_{1} B_{2}}\left(\phi_{A_{1} A_{2} A^{\prime}}\right)$. 
3) Entanglement-formation lower bound

$$
\left.C_{\mathrm{Q}}\left(\mathscr{N}^{\text {relay }}\right) \geq \max _{\left|\phi_{A_{1} A_{2} A^{\prime}}\right\rangle, \mathscr{F}_{B_{1} \rightarrow \widehat{B}_{1}}: E_{F}\left(\rho_{\widehat{B}_{1} A B_{2} E}\right) \leq \mathrm{C}_{Q, 12}} I\left(A_{2}\right\rangle \widehat{B}_{1} B_{2}\right)_{\phi}
$$

with $\left|\phi_{A B_{1} B_{2} E}\right\rangle=U_{A^{\prime} \rightarrow B_{1} B_{2} E}^{\mathscr{N}}\left|\phi_{A A^{\prime}}\right\rangle, \rho_{A \widehat{B}_{1} B_{2} E}=\mathscr{F}_{B_{1} \rightarrow \widehat{B}_{1}}\left(\phi_{A B_{1} B_{2} E}\right)$, where $E_{F}\left(\rho_{\widehat{B}_{1} A B_{2} E}\right)$ is the entanglement of formation with respect to the bipartition $\widehat{B}_{1} \mid A B_{2} E$.

The proof of the cutset upper bound follows the same considerations as in Subsection V B, and it is thus omitted (see (40) and (41)). The decode-forward lower bound in Theorem 5 above is obtained as an immediate consequence of Theorem 3, taking $Q_{1}=0$. The rate in (44) can be achieved by using the conferencing link to simulate the channel $\mathscr{F}_{B_{1} \rightarrow \widehat{B}_{1}}$. Based on the results of Berta et al. (Ref. 98, Theo. 12), this can be achieved if the capacity of the conference link is higher that the entanglement of formation with respect to the bipartition $\widehat{B}_{1} \mid A B_{2} E$, i.e. $C_{Q, 12} \geq$ $E_{F}\left(\rho_{\widehat{B}_{1} A B_{2} E}\right)$. Then, Bob 2 can decode $\rho_{\hat{B}_{1}^{n} B_{2}^{n}}$, which is $\varepsilon_{n}$-close in trace distance to $\rho_{\hat{B}_{1}^{n} B_{2}^{n}} \equiv$ $\widehat{\mathscr{N}}_{A \rightarrow \hat{B}_{1} B_{2}}\left(\rho_{A^{\prime n}}\right)$, where $\varepsilon_{n}$ tends to zero as $n \rightarrow \infty$, with $\widehat{\mathscr{N}}_{A \rightarrow \hat{B}_{1} B_{2}} \triangleq \mathscr{F}_{B_{1} \rightarrow \widehat{B}_{1}} \circ \mathscr{N}_{A \rightarrow B_{1} B_{2}}$.

Remark 9. Recall from the beginning of Sec. V that we view Alice, Bob 1, and Bob 2 as the sender, repeater, and destination receiver. In other words, the repeater is the quantum version of a relay. As we also consider direct transmission to the destination receiver (Bob 2), our results show the tradeoff between repeaterless communication and relaying information through the repeater. In particular, in the decode-forward lower bound (43) (see part 2 of Theorem 5), the term $\left.I\left(A_{2}\right\rangle B_{2}\right)_{\rho}$ corresponds to repeaterless communication, while $\left.\min \left(I\left(A_{1}\right\rangle B_{1}\right)_{\rho}, C_{Q, 12}\right)$ corresponds to quantum transmission via the repeater.

Remark 10. Intuitively, the decode-forward lower bound has the interpretation of a bottleneck flow. Specifically, as mentioned in the previous remark, the term $\left.\min \left(I\left(A_{1}\right\rangle B_{1}\right)_{\rho}, C_{Q, 12}\right)$ in the decodeforward lower bound (43) is associated with the information rate via the repeater. Due to the serial connection between the sender-repeater link $A \rightarrow B_{1}$ with the repeater-receiver link $B_{1} \rightarrow B_{2}$, the throughput is dictated by the smaller rate (see Fig. 4). A similar behavior was observed by Smolin et al. ${ }^{93}$ for a quantum channel with environment assistance of a classical relay (see Ref. 93, Th. 8, and Refs. 92 and 94 as well).

\section{ENTANGLED DECODERS}

In this section, we consider a broadcast channel where the decoders share entanglement resources between them (see Fig. 1). Given the recent results by Leditzky et al. ${ }^{45}$ on the multiple 
access channel, it may be tempting to think that the dual property holds for the broadcast channel and that entanglement between decoders can increase achievable rates of classical communication. We observe that this is not the case. Nevertheless, given a quantum conferencing link of capacity $C_{Q, 12}$, Receiver 1 can send conferencing messages to Receiver 2 at a rate $2 \cdot C_{Q, 12}$ using the super-dense coding protocol. Further details are given below.

First, consider a quantum broadcast channel $\mathscr{N}_{A \rightarrow B_{1} B_{2}}$ without conferencing, given entanglement resources shared between the decoders, as illustrated in Fig. 1. We show that the classical capacity region is the same as without the entanglement resources. Indeed, suppose that Alice chooses $m_{0}$ and $m_{1}$ uniformly at random, and prepares an input state $\rho_{A^{n}}^{m_{0}, m_{1}}$. After Alice sends the systems $A^{n}$ through the channel, the output state is $\rho_{B_{1}^{n} B_{2}^{n}} \otimes \Psi_{S_{B_{1}} S_{B_{2}}}$, where $S_{B_{1}}$ and $S_{B_{2}}$ are the entangled systems of Bob 1 and Bob 2, respectively, and $\rho_{B_{1}^{n} B_{2}^{n}}=\frac{1}{2^{n\left(R_{0}+R_{1}\right)}} \sum_{m_{0}, m_{1}} \mathscr{N}_{A^{n} \rightarrow B_{1}^{n} B_{2}^{n}}\left(\rho_{A^{n}}^{m_{0}, m_{1}}\right)$. Then, Bob 1 performs a decoding POVM $\Lambda_{B_{1}^{n} S_{B_{1}}}^{m_{0}, m_{1}}$, and Bob 2 performs a decoding POVM $\Lambda_{B_{2}^{n} S_{B_{2}}}^{m_{0}}$. Consider a sequence of codes $\left(\mathscr{F}_{n}, \Lambda_{n}, \Gamma_{n}\right)$ such that the average probability of error tends to zero, hence the error probabilities $\operatorname{Pr}\left(\widehat{M}_{0} \neq M_{0}\right), \operatorname{Pr}\left(\left(\widehat{M}_{0}, \widehat{M}_{1}\right) \neq\left(M_{0}, M_{1}\right)\right), \operatorname{Pr}\left(\widehat{M}_{1} \neq M_{1} \mid M_{0}\right)$ are bounded by some $\alpha_{n}$ which tends to zero as $n \rightarrow \infty$. By Fano's inequality, it follows that

$$
\begin{array}{r}
H\left(M_{0} \mid \widetilde{M}_{0}\right) \leq n \varepsilon_{n} \\
H\left(M_{0}, M_{1} \mid \widehat{M}_{0}, \widehat{M}_{1}\right) \leq n \varepsilon_{n}^{\prime} \\
H\left(M_{1} \mid \widehat{M}_{1}, M_{0}\right) \leq n \varepsilon_{n}^{\prime \prime}
\end{array}
$$

where $\varepsilon_{n}, \varepsilon_{n}^{\prime}, \varepsilon_{n}^{\prime \prime}$ tend to zero as $n \rightarrow \infty$. Hence,

$$
\begin{aligned}
n R_{0}=H\left(M_{0}\right)=I\left(M_{0} ; \widetilde{M}_{0}\right)_{\rho}+H\left(M_{0} \mid \widetilde{M}_{0}\right) & \leq I\left(M_{0} ; \widetilde{M}_{0}\right)_{\rho}+n \varepsilon_{n} \\
& \leq I\left(M_{0} ; B_{2}^{n} S_{B_{2}}\right)_{\rho}+n \varepsilon_{n}=I\left(M_{0} ; B_{2}^{n}\right)_{\rho}
\end{aligned}
$$

where the second inequality follows from the Holevo bound (see Ref. 33, Theo. 12.1), and the last inequality holds as $S_{B_{1}} S_{B_{2}}$ are in a product state with $M_{0}, M_{1}, B_{1}^{n}, B_{2}^{n}$. Similarly,

$$
\begin{aligned}
n\left(R_{0}+R_{1}\right) & \leq I\left(M_{0}, M_{1} ; B_{1}^{n}\right)_{\rho}+n \varepsilon_{n}^{\prime} \\
n R_{1} & \leq I\left(M_{1} ; B_{1}^{n} \mid M_{0}\right)_{\rho}+n \varepsilon_{n}^{\prime} .
\end{aligned}
$$

as without entanglement resources.

We observe that the this property can be extended to any pair of non-signaling correlated resources that are shared between the decoders. Specifically, suppose that Bob 1 and Bob 2 have 
random elements $\beta_{1}$ and $\beta_{2}$, that follow a non-signaling correlation $\left(\beta_{1}, \beta_{2}\right) \sim p\left(b_{1}, b_{2} \mid x_{1}, x_{2}\right)$, such that

$$
\begin{aligned}
& \sum_{b_{2}} p\left(b_{1}, b_{2} \mid x_{1}, x_{2}\right)=p\left(b_{1} \mid x_{1}\right) \\
& \sum_{b_{1}} p\left(b_{1}, b_{2} \mid x_{1}, x_{2}\right)=p\left(b_{2} \mid x_{2}\right)
\end{aligned}
$$

for some $x_{1}$ and $x_{2}$. We think of $\beta_{k}$ as the measurement outcome of Bob $k$, while $x_{k}$ is his choice of measurement, for $k=1,2$. The derivation of the outer bound above boils down to the fact that Bob 1 and Bob 2's resources are uncorrelated with the message and the channel outputs. Thus, the capacity region of the broadcast channel with non-signaling correlated resources between the decoders is the same as without those resources.

On the other hand, given a quantum conferencing link of capacity $C_{Q, 12}$, the classical capacity region with entanglement between the decoders is given by the regularization of the region in (23), taking $C_{12}=2 \cdot C_{Q, 12}$. Achievability follows by using the super-dense coding protocol ${ }^{38}$ to send classical conferencing messages from Bob 1 to Bob 2. As for the converse proof, consider a coding scheme where Bob 1 performs a decoding POVM $\Lambda_{B_{1}^{n} S_{B_{1}}}^{m_{0}, m_{1} g}$, sends $g$ to Bob 2 using conferencing, and Bob 2 chooses a POVM $\Gamma_{B_{2}^{n} S_{B_{2}} \mid g}^{m_{0}}$ accordingly. By the same considerations as in the derivation above,

$$
\begin{aligned}
n R_{0} & \leq I\left(M_{0} ; \widetilde{M}_{0}\right)_{\rho}+n \varepsilon_{n} \\
& \leq I\left(M_{0} ; B_{2}^{n} S_{B_{2}} G^{\prime}\right)_{\rho}+n \varepsilon_{n} \\
& =I\left(M_{0} ; B_{2}^{n} S_{B_{2}}\right)_{\rho}+I\left(M_{0} ; G^{\prime} \mid B_{2}^{n} S_{B_{2}}\right)_{\rho}+n \varepsilon_{n} \\
& \leq I\left(M_{0} ; B_{2}^{n}\right)_{\rho}+2 \cdot n C_{Q, 12}+n \varepsilon_{n}
\end{aligned}
$$

where the second inequality follows from the Holevo bound (see Ref. 33, Theo. 12.1), the equality is due to chain rule for the quantum mutual information, and the last inequality holds because $I\left(M_{0} ; B_{2}^{n} S_{B_{2}}\right)_{\rho}=I\left(M_{0} ; B_{2}^{n}\right)_{\rho}$ as $S_{B_{1}} S_{B_{2}}$ are in a product state with $M_{0}, M_{1}, B_{1}^{n}, B_{2}^{n}$, and since $I\left(M_{0} ; G^{\prime} \mid B_{2}^{n} S_{B_{2}}\right)_{\rho} \leq 2 H\left(G^{\prime}\right)_{\rho} \leq 2 \cdot n C_{Q, 12}$ (see Ref. 36, Sec. 11.6). As the bounds (49)-(50) hold by similar arguments, the proof follows.

We conclude that entanglement between the decoders cannot enlarge the capacity region of the classical broadcast channel without conferencing. By similar considerations, the same property holds for a broadcast channel with classical conferencing as well, and more generally, for any pair of non-signaling correlated resources. Yet, entanglement resources between the decoders 
double the conferencing rate when a quantum conferencing link is available. Further observations and a comparison with the multiple access channel are provided in the discussion section, in Subsec. VII C.

\section{SUMMARY AND DISCUSSION}

We have considered the quantum broadcast channel $\mathscr{N}_{A \rightarrow B_{1} B_{2}}$ in different settings of cooperation between the decoders. Using those settings, we provided an information-theoretic framework for quantum repeaters.

\section{A. Conferencing}

The first form of cooperation that we considered is classical conferencing, where Receiver 1 can send classical messages to Receiver 2. We provided a regularized characterization for the classical capacity region of the quantum broadcast channel with classical conferencing, and a single-letter formula for Hadamard broadcast channels. Next, we considered quantum conferencing, where Receiver 1 can teleport a quantum state to Receiver 2. We developed inner and outer bounds on the quantum capacity region with quantum conferencing, characterizing the tradeoff between the communication rates $Q_{1}$ and $Q_{2}$ to Receiver 1 and Reciever 2, respectively, as well as the conferencing capacity $\mathrm{C}_{Q, 12}$.

Quantum communication is also referred to as entanglement transmission and can be extended to strong subspace transmission ${ }^{31,111}$. In this task, Alice and Charlie share a pure entangled state $\left|\psi_{M_{1} M_{2} C}\right\rangle$, and at the end of the communication protocol, Bob 1, Bob 2, and Charlie share a state $\approx\left|\Phi_{\widehat{M}_{1} \widehat{M}_{2} C}\right\rangle$ up to an $\varepsilon$-error. In the absence of entanglement resources between the decoders, quantum communication over the broadcast channel can generate such entanglement by choosing the quantum message state to be $\left|\Phi_{M_{1} M_{2}}\right\rangle$.

Quantum communication can also be used for the purpose of entanglement generation ${ }^{28,31}$. We note that by the monogamy property of quantum entanglement ${ }^{79}$, Alice cannot generate a maximally entangled state with both Bob 1 and Bob 2 simultaneously. Nevertheless, different forms of entanglement can be generated. In particular, Alice can generate a GHZ state with Bob 1 and Bob 2 (Ref. 55, Sec. IV), using $\left|\psi_{\bar{A} M_{1} M_{2}}\right\rangle=\frac{1}{\sqrt{d}} \sum_{x=1}^{d}|x\rangle \otimes|x\rangle \otimes|x\rangle$. Alternatively, she can 
generate two entangled pairs. Suppose that Alice has another pair of system $\bar{A}_{1}, \bar{A}_{2}$ in the state

$$
\left|\psi_{\bar{A}_{1} M_{1} \bar{A}_{1} M_{2}}\right\rangle=\left|\Phi_{\bar{A}_{1} M_{1}}\right\rangle \otimes\left|\Phi_{\bar{A}_{2} M_{2}}\right\rangle .
$$

Then, at the end of the quantum communication protocol, Alice shares the entangled states $\approx$ $\left|\Phi_{\bar{A}_{1} \widehat{M}_{1}}\right\rangle$ with Bob 1 and $\approx\left|\Phi_{\bar{A}_{2} \widehat{M}_{2}}\right\rangle$ with Bob 2.

The case where Receiver 1 is not required to recover information, i.e. $Q_{1}=0$, and its sole purpose is to help the transmission to Receiver 2, reduces to the quantum primitive relay channel, for which the decode-forward lower bound and cutset upper bound follow as a consequence. In addition, we established an entanglement-formation lower bound, where a virtual channel is simulated through the conference link, following the results of Berta et al. ${ }^{98}$ on quantum channel simulation.

\section{B. Quantum Repeaters}

The quantum conferencing setting is intimately related to quantum repeaters, as the sender, Receiver 1, and Receiver 2 can be viewed as the transmitter, the repeater, and the destination receiver, respectively, in the repeater model. In particular, the sender can employ quantum communication to Receiver 1 (the repeater) in order to prepare a maximally entangled pair $\left|\Phi_{A B_{1}}\right\rangle$, which consists of $n Q_{1}$ entangled bits (ebits).

Given entanglement between the receivers, we also have a maximally entangled pair $\left|\Phi_{B_{1}^{\prime} B_{2}^{\prime}}\right\rangle$, which consists of $n C_{Q, 12}=\frac{1}{2} n C_{12}$ ebits, shared between the repeater and the destination receiver, where $C_{12}$ is the classical conferencing link. Then, the repeater can swap his entanglement by using the classical conferencing link to teleport the state of $B_{1}^{\prime}$ onto $B_{2}$ thus swapping the entanglement such that $A$ and $B_{2}$ are now entangled. This requires the classical conferencing rate to be at least twice the information transmission rate to $B_{2}$.

Hence our results provide an information-theoretic analysis characterizing the achievable rates of ebits that can be generated in each stage. As we have also considered direct transmission to the destination receiver, our results reflect the tradeoff between repeaterless communication and relaying qubits using the repeater as well (see Rem. 9).

Intuitively, the communication via the repeater gives rise to a bottleneck effect. That is, due to the serial connection between the sender-repeater link $A \rightarrow B_{1}$ with the repeater-receiver link $B_{1} \rightarrow B_{2}$, the throughput is dictated by the smaller rate (see Fig. 4). Indeed, the term in the decode-forward formula (43) that is associated with communication via the repeater involves a 
minimum between the coherent information $\left.I\left(A_{1}\right\rangle B_{1}\right) \rho$ and the conferencing link capacity $C_{Q, 12}$ (see Rem. 10).

\section{BC-MAC Duality}

The duality between the broadcast channel and the multiple access channel (BC-MAC duality) is a well-known property in the study of Gaussian multiple-input multiple-output (MIMO) channels ${ }^{104-106}$. Based on the reciprocity property ${ }^{114}$, the capacity remains unchanged when the role of the transmitters and receivers is interchanged ${ }^{104-106}$ (see also Ref. 115, Lemm. 9.2). In the scalar case, this means that the capacity region of the Gaussian broadcast channel,

$$
\begin{aligned}
& Y_{1}=h_{1} X+Z_{1} \\
& Y_{2}=h_{2} X+Z_{2}
\end{aligned}
$$

subject to a power constraint $\frac{1}{n} \sum_{i=1}^{n} x_{i}^{2} \leq P$, is exactly the same as the capacity region of the Gaussian multiple access channel,

$$
Y=h_{1} X_{1}+h_{2} X_{2}+Z
$$

subject to a total-power constraint $\frac{1}{n} \sum_{i=1}^{n}\left(x_{1, i}^{2}+x_{2, i}^{2}\right) \leq P$, with normalized Gaussian noise $Z, Z_{1}$, $Z_{2} \sim \mathscr{N}(0,1)$. As the multiple access channel and broadcast channel are useful models for uplink and downlink transmission in cellular communication, this behavior is also referred to as uplinkdownlink duality. Duality properties have also been shown for beamforming strategies ${ }^{116,117}$.

Our result demonstrates the limitations of the duality between the broadcast channel and the multiple access channel. Leditzky et al. ${ }^{45}$ considered a classical multiple access channel $P_{Y \mid X_{1} X_{2}}$, with two senders and a single receiver, when the encoders share entanglement resources, as illustrated in Fig. 5. The MAC in Ref. 45 is defined in terms of a pseudo-telepathy game ${ }^{46}$, for which quantum strategies guarantee a certain win and outperform classical strategies. They showed achievability of a sum-rate $R_{1}+R_{2}$ that exceeds the sum-rate capacity of this channel without entanglement. In principle, one could mirror the model ( $c f$. Fig. 1 and Fig. 5), and consider a broadcast channel $P_{Y_{1}^{\prime} Y_{2}^{\prime} \mid X^{\prime}}$, where $X^{\prime} \equiv Y, Y_{1} \equiv X_{1}$, and $Y_{2}^{\prime} \equiv X_{2}$, according to the a posteriori probability distribution $P_{X_{1} X_{2} \mid Y}$ given some input distribution $p_{X_{1}, X_{2}}$. Specifically, the derivation in Ref. 45 is for the magic square game ${ }^{46}$, which is highly symmetric. Hence, it can be shown that the sum-rate capacity of the multiple access channel $P_{Y \mid X_{1} X_{2}}$ and the broadcast channel $P_{Y_{1}^{\prime} Y_{2}^{\prime} \mid X^{\prime}}$, without 
entanglement resources, are the same. Nevertheless, we cannot use the entanglement cooperation in the same manner, as the decoding strategy does not affect the channel. Intuitively, encoding using quantum game strategies for the multiple access channel inserts quantum correlations into the channel. On the other hand, in the broadcast setting, the entangled resources of the decoders are not correlated with the channel inputs or outputs. This observation explains the asymmetry with regard to entanglement cooperation, and more generally, for any pair of non-signaling correlated resources that are shared between the decoders. Therefore, our result reveals a fundamental asymmetry and demonstrates the limitations of the duality between the broadcast channel and the multiple access channel.

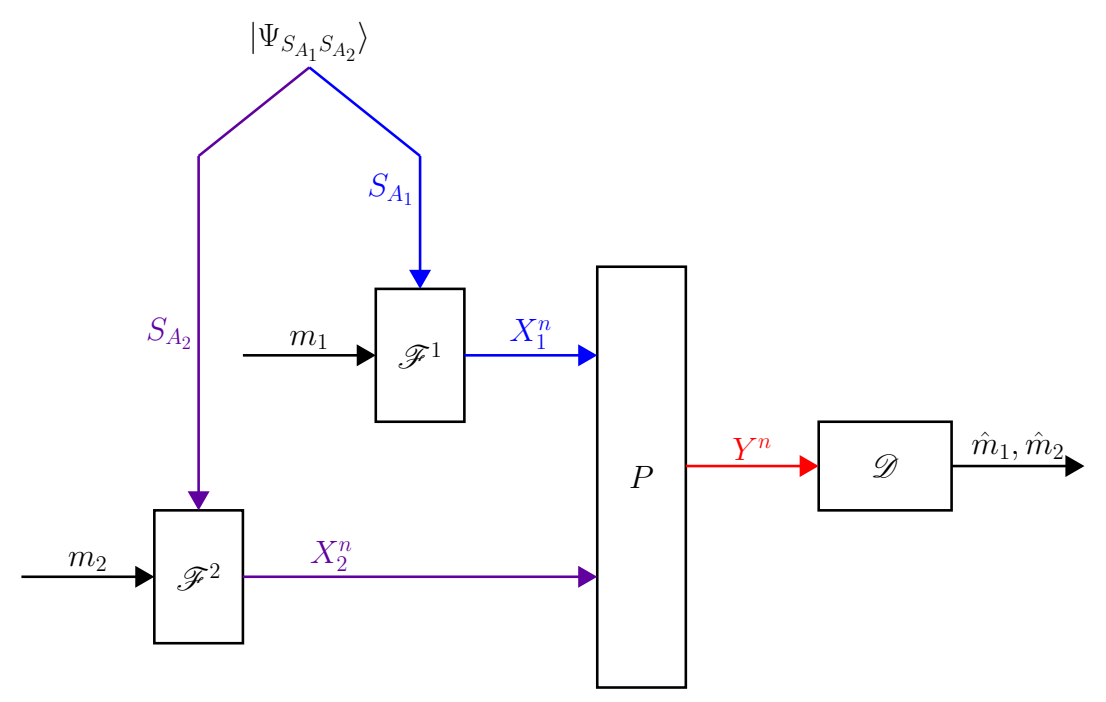

FIG. 5. Classical coding for a classical multiple access channel $P_{Y \mid X_{1} X_{2}}$ with shared entanglement between the encoders. The systems of Alice 1, Alice 2, and Bob are marked in blue, purple and red, respectively. Alice 1 and Alice 2 share entanglement resources in the systems $S_{A_{1}}$ and $S_{A_{2}}$, respectively. For $k=1,2$, Alice $k$ encodes the message $m_{k}$ by applying an encoding map $\mathscr{F}_{M_{k} \rightarrow x_{k}^{n}}$ to the register $M_{k}$ which stores the respective message. Then, she transmits $X_{k}^{n}$ over the multiple access channel. Bob receives the channel output sequence $Y^{n}$, and estimates the messages using a decoding map $\mathscr{D}_{Y^{n} \rightarrow \widehat{M}_{1} \widehat{M}_{2}}$. Leditzky et al. ${ }^{45}$ showed that using the entanglement resources, $S_{A_{1}}$ and $S_{A_{2}}$, can strictly increase the achievable rates. We have shown that for the broadcast dual in Fig. 1, entanglement resources cannot increase the achievable rates. 


\section{ACKNOWLEDGMENTS}

Uzi Pereg, Christian Deppe, and Holger Boche were supported by the Bundesministerium für Bildung und Forschung (BMBF) through Grants 16KIS0856 (Pereg, Deppe), 16KIS0858 (Boche), and the Israel CHE Fellowship for Quantum Science and Technology (Pereg). This work of H. Boche was also supported in part by the German Research Foundation (DFG), within the Gottfried Wilhelm Leibniz Prize under Grant BO 1734/20-1 and within Germany's Excellence Strategy EXC-2111-390814868.

\section{DATA AVAILABILITY}

The data that supports the findings of this study are available within the article.

\section{Appendix A: Proof of Lemma 1}

To bound the alphabet size of the random variables $X_{0}$ and $X_{1}$, we use the Fenchel-EgglestonCarathéodory lemma ${ }^{118}$ and similar arguments as in Refs. 75 and 119 . Let

$$
\begin{aligned}
& L_{0}=\left|\mathscr{H}_{A}\right|^{2}+2 \\
& L_{1}=L_{0}\left|\mathscr{H}_{A}\right|^{2}+1 .
\end{aligned}
$$

First, fix $p_{X_{1} \mid X_{0}}\left(x_{1} \mid x_{0}\right)$, and consider the ensemble $\left\{p_{X_{0}}\left(x_{0}\right) p_{X_{1} \mid X_{0}}\left(x_{1} \mid x_{0}\right), \theta_{A}^{x_{0}, x_{1}}\right\}$. Every pure state $\theta_{A}=\left|\phi_{A}\right\rangle\left\langle\phi_{A}\right|$ has a unique parametric representation $u\left(\theta_{A}\right)$ of dimension $\left|\mathscr{H}_{A}\right|^{2}-1$. Then, define a map $f_{0}: \mathscr{X}_{0} \rightarrow \mathbb{R}^{L_{0}}$ by

$$
f_{0}\left(x_{0}\right)=\left(u\left(\rho_{A}^{x_{0}}\right), H\left(B_{2} \mid X_{0}=x_{0}\right), H\left(B_{1} \mid X_{0}=x_{0}\right)_{\rho}, H\left(B_{1} \mid X_{0}=x_{0}, X_{1}\right)\right)
$$

where $\rho_{A}^{x_{0}}=\sum_{x_{1}} p_{X_{1} \mid X_{0}}\left(x_{1} \mid x_{0}\right) \theta_{A}^{x_{0}, x_{1}}$. The map $f_{0}$ can be extended to a map that acts on probability distributions as follows,

$$
F_{0}: p_{X_{0}} \mapsto \sum_{x_{0} \in \mathscr{X}_{0}} p_{X_{0}}\left(x_{0}\right) f_{0}\left(x_{0}\right)=\left(u\left(\rho_{A}\right), H\left(B_{2} \mid X_{0}\right), H\left(B_{1} \mid X_{0}\right)_{\rho}, H\left(B_{1} \mid X_{0}, X_{1}\right)\right)
$$

where $\rho_{A}=\sum_{x_{0}} p_{X_{0}}\left(x_{0}\right) \rho_{A}^{x_{0}}$. According to the Fenchel-Eggleston-Carathéodory lemma ${ }^{118}$, any point in the convex closure of a connected compact set within $\mathbb{R}^{d}$ belongs to the convex hull of $d$ points in the set. Since the map $F_{0}$ is linear, it maps the set of distributions on $\mathscr{X}_{0}$ to a 
connected compact set in $\mathbb{R}^{L_{0}}$, where $L_{0}=\left(\left|\mathscr{H}_{A}\right|^{2}-1\right)+1+1=\left|\mathscr{H}_{A}\right|^{2}+1$ as defined in (A1). Thus, for every $p_{X_{0}}$, there exists a probability distribution $p_{\bar{X}_{0}}$ on a subset $\overline{\mathscr{X}}_{0} \subseteq \mathscr{X}_{0}$ of size $L_{0}$, such that $F_{0}\left(p_{\bar{X}_{0}}\right)=F_{0}\left(p_{X_{0}}\right)$. We deduce that alphabet size can be restricted to $\left|\mathscr{X}_{0}\right| \leq L_{0}$, while preserving $\rho_{A}$ and $\rho_{B_{1} B_{2}} \equiv \mathscr{N}_{A \rightarrow B_{1} B_{2}}\left(\rho_{A}\right) ; I\left(X_{0} ; B_{2}\right)_{\rho}=H\left(B_{2}\right)_{\rho}-H\left(B_{2} \mid X_{0}\right)_{\rho}, I\left(X_{1} ; B_{1} \mid X_{0}\right)_{\rho}=$ $H\left(B_{1} \mid X_{0}\right)_{\rho}-H\left(B_{1} \mid X_{0}, X_{1}\right)_{\rho}$; and $I\left(X_{0}, X_{1} ; B_{1}\right)_{\rho}=H\left(B_{1}\right)_{\rho}-H\left(B_{1} \mid X_{0}, X_{1}\right)_{\rho}$.

We move to the alphabet size of $X_{1}$. Fix $p_{X_{0} \mid X_{1}}$, where

$$
p_{X_{0} \mid X_{1}}\left(x_{0} \mid x_{1}\right) \equiv \frac{p_{X_{0}}\left(x_{0}\right) p_{X_{1} \mid X_{0}}\left(x_{1} \mid x_{0}\right)}{\sum_{x_{0}^{\prime} \in \mathscr{X}_{0}} p_{X_{0}}\left(x_{0}^{\prime}\right) p_{X_{1} \mid X_{0}}\left(x_{1} \mid x_{0}^{\prime}\right)} .
$$

Define the map $f_{1}: \mathscr{X}_{1} \rightarrow \mathbb{R}^{L_{1}}$ by

$$
f_{1}\left(x_{1}\right)=\left(p_{X_{0} \mid X_{1}}\left(\cdot \mid x_{1}\right),\left(u\left(\rho_{A}^{x_{0}, x_{1}}\right)\right)_{x_{0} \in \mathscr{X}_{0}}, H\left(B_{1} \mid X_{0}, X_{1}=x_{1}\right)_{\rho}\right)
$$

where $\rho_{A}^{x_{1}}=\sum_{x_{0}} p_{X_{0} \mid X_{1}}\left(x_{0} \mid x_{1}\right) \theta_{A}^{x_{0}, x_{1}}$. Now, the extended map is

$$
F_{1}: p_{X_{1}} \mapsto \sum_{x_{1} \in \mathscr{X}_{1}} p_{X_{1}}\left(x_{1}\right) f_{1}\left(x_{1}\right)=\left(p_{X_{0}},\left(u\left(\rho_{A}^{x_{0}}\right)\right)_{x_{0} \in \mathscr{X}_{0}}, H\left(B_{1} \mid X_{0}, X_{1}\right)_{\rho}\right)
$$

By the Fenchel-Eggleston-Carathéodory lemma ${ }^{118}$, for every $p_{X_{1}}$, there exists $p_{\bar{X}_{1}}$ on a subset $\overline{\mathscr{X}}_{1} \subseteq \mathscr{X}_{1}$ of size $\left(\left|\mathscr{H}_{A}\right|^{2}-1\right) L_{0}+2 \leq L_{1}$ (see (A1)), such that $F_{1}\left(p_{\bar{X}_{1}}\right)=F_{1}\left(p_{X_{1}}\right)$. We deduce that alphabet size can be restricted to $\left|\mathscr{X}_{1}\right| \leq L_{1}$, while preserving $\rho_{A}^{x_{0}}, \rho_{A}$ and $\rho_{B_{1} B_{2}}^{x_{0}} \equiv \mathscr{N}_{A \rightarrow B_{1} B_{2}}\left(\rho_{A}^{x_{0}}\right)$, $\rho_{B_{1} B_{2}} \equiv \mathscr{N}_{A \rightarrow B_{1} B_{2}}\left(\rho_{A}\right) ; I\left(X_{1} ; B_{1} \mid X_{0}\right)_{\rho}=H\left(B_{1} \mid X_{0}\right)_{\rho}-H\left(B_{1} \mid X_{0}, X_{1}\right)_{\rho} ;$ and $I\left(X_{0}, X_{1} ; B_{1}\right)_{\rho}=H\left(B_{1}\right)_{\rho}$ $-H\left(B_{1} \mid X_{0}, X_{1}\right) \rho$.

\section{Appendix B: Proof of Theorem 2}

Consider a quantum broadcast channel $\mathscr{N}_{A \rightarrow B_{1} B_{2}}$ with classical conferencing link of capacity $C_{12}$. The proof extends techniques that were used in a previous work by the first author ${ }^{119,120}$.

\section{Achievability Proof}

We show that for every $\zeta_{0}, \zeta_{1}, \varepsilon_{0}>0$, there exists a $\left(2^{n\left(R_{0}-\zeta_{0}\right)}, 2^{n\left(R_{1}-\zeta_{1}\right)}, n, \varepsilon_{0}\right)$ code for $\mathscr{N}_{A \rightarrow B_{1} B_{2}}$ with conferencing and degraded message sets, provided that $\left(R_{0}, R_{1}\right) \in \mathscr{R}_{\mathrm{Cl}}(\mathscr{N})$. To prove achievability, we extend the classical superposition coding with binning technique to the quantum setting, and then apply the quantum packing lemma. Similar observations as in Refs. 
Quantum Broadcast Channels with Cooperating Decoders

119 and 120 are used as well. Let $\left\{p_{X_{0}}\left(x_{0}\right) p_{X_{1} \mid X_{0}}\left(x_{1} \mid x_{0}\right), \theta_{A}^{x_{0}, x_{1}}\right\}$ be a given ensemble, and define

$$
\begin{aligned}
\rho_{B_{1}, B_{2}}^{x_{0}, x_{1}} & \equiv \mathscr{N}_{A \rightarrow B_{1} B_{2}}\left(\theta_{A}^{x_{0}, x_{1}}\right) \\
\sigma_{B_{2}}^{x_{0}} & \equiv \sum_{x_{1}^{\prime} \in \mathscr{X}_{1}} p_{X_{1} \mid X_{0}}\left(x_{1}^{\prime} \mid x_{0}\right) \rho_{B_{2}}^{x_{0}, x_{1}}
\end{aligned}
$$

for $\left(x_{0}, x_{1}\right) \in \mathscr{X}_{0} \times \mathscr{X}_{1}$, where $\rho_{B_{2}}^{x_{0}, x_{1}}$ is the reduced state of $\rho_{B_{1}, B_{2}}^{x_{0}, x_{1}}$.

Standard method-of-types concepts are defined as in Refs. 36 and 119. We briefly introduce the notation and basic properties while the detailed definitions can be found in Ref. 119, Sec. III. In particular, given a density operator $\rho=\sum_{x} p_{X}(x)|x\rangle\langle x|$ on the Hilbert space $\mathscr{H}_{A}$, we let $\mathscr{A}^{\delta}\left(p_{X}\right)$ denote the $\delta$-typical set that is associated with $p_{X}$, and $\Pi_{A^{n}}^{\delta}(\rho)$ the projector onto the corresponding subspace. The following inequalities follow from well-known properties of $\delta$-typical sets ${ }^{33}$,

$$
\begin{aligned}
\operatorname{Tr}\left(\Pi^{\delta}(\rho) \rho^{\otimes n}\right) & \geq 1-\varepsilon \\
2^{-n(H(\rho)+c \delta)} \Pi^{\delta}(\rho) & \preceq \Pi^{\delta}(\rho) \rho^{\otimes n} \Pi^{\delta}(\rho) \preceq 2^{-n(H(\rho)-c \delta)} \\
\operatorname{Tr}\left(\Pi^{\delta}(\rho)\right) & \leq 2^{n(H(\rho)+c \delta)}
\end{aligned}
$$

where $c>0$ is a constant. Furthermore, for $\sigma_{B}=\sum_{x} p_{X}(x) \rho_{B}^{x}$, let $\Pi_{B^{n}}^{\delta}\left(\sigma_{B} \mid x^{n}\right)$ denote the projector corresponding to the conditional $\delta$-typical set given the sequence $x^{n}$. Similarly ${ }^{36}$,

$$
\begin{aligned}
\operatorname{Tr}\left(\Pi^{\delta}\left(\sigma_{B} \mid x^{n}\right) \rho_{B^{n}}^{x^{n}}\right) & \geq 1-\varepsilon^{\prime} \\
2^{-n\left(H\left(B \mid X^{\prime}\right)_{\sigma}+c^{\prime} \delta\right)} \Pi^{\delta}\left(\sigma_{B} \mid x^{n}\right) & \preceq \Pi^{\delta}\left(\sigma_{B} \mid x^{n}\right) \rho_{B^{n}}^{x^{n}} \Pi^{\delta}\left(\sigma_{B} \mid x^{n}\right) \preceq 2^{-n\left(H\left(B \mid X^{\prime}\right)_{\sigma}-c^{\prime} \delta\right)} \\
\operatorname{Tr}\left(\Pi^{\delta}\left(\sigma_{B} \mid x^{n}\right)\right) & \leq 2^{n\left(H\left(B \mid X^{\prime}\right)_{\sigma}+c^{\prime} \delta\right)}
\end{aligned}
$$

where $c^{\prime}>0$ is a constant, $\rho_{B^{n}}^{x^{n}}=\bigotimes_{i=1}^{n} \rho_{B_{i}}^{x_{i}}$, and the classical random variable $X^{\prime}$ is distributed according to the type of $x^{n}$. If $x^{n} \in \mathscr{A}^{\delta}\left(p_{X}\right)$, then

$$
\operatorname{Tr}\left(\Pi^{\delta}\left(\sigma_{B}\right) \rho_{B^{n}}^{x^{n}}\right) \geq 1-\varepsilon^{\prime}
$$

as well (see Ref. 36, Property 15.2.7). We note that the conditional entropy in the bounds above can also be expressed as $H\left(B \mid X^{\prime}\right)_{\sigma}=\frac{1}{n} H\left(B^{n} \mid X^{n}=x^{n}\right)_{\sigma} \equiv \frac{1}{n} H\left(B^{n}\right)_{\rho^{x^{n}}}$.

The code construction, encoding and decoding procedures are described below.

Classical Code Construction: Select $2^{n R_{0}}$ independent sequences $x_{0}^{n}\left(m_{0}\right), m_{0} \in\left[1: 2^{n R_{0}}\right]$, at random according to $\prod_{i=1}^{n} p_{X_{0}}\left(x_{0, i}\right)$. For every $m_{0} \in\left[1: 2^{n R_{0}}\right]$, select $2^{n R_{1}}$ conditionally independent sequences $x_{1}^{n}\left(m_{0}, m_{1}\right), m_{1} \in\left[1: 2^{n R_{1}}\right]$, at random according to $\prod_{i=1}^{n} p_{X_{1} \mid X_{0}}\left(x_{1, i} \mid x_{0, i}\left(m_{0}\right)\right.$. Partition the set of indices $\left[1: 2^{n R_{0}}\right]$ into $2^{n C_{12}}$ bins of equal size,

$$
\mathscr{B}(g)=\left[(g-1) 2^{n\left(R_{0}-\mathrm{C}_{12}\right)}: g 2^{n\left(R_{0}-\mathrm{C}_{12}\right)}\right]
$$


Quantum Broadcast Channels with Cooperating Decoders

for $g \in\left[1: 2^{\mathrm{C}_{12}}\right]$.

Encoding: To send the message pair $\left(m_{0}, m_{1}\right)$, Alice prepares $\rho_{A^{n}}=\bigotimes_{i=1}^{n} \rho_{A}^{x_{1, i}\left(m_{0}, m_{1}\right)}$ and sends the block $A^{n}$. The resulting output state is

$$
\rho_{B_{1}^{n} B_{2}^{n}}=\bigotimes_{i=1}^{n} \rho_{B}^{x_{0, i}\left(m_{0}\right), x_{1, i}\left(m_{0}, m_{1}\right)}
$$

Decoding: Bob 1 receives the systems $B_{1}^{n}$ and decodes as follows.

(i) Decode $\hat{m}_{0}$ by applying a POVM $\left\{\Lambda_{m_{0}}^{0}\right\}_{m_{0} \in\left[1: 2^{n R_{0}}\right]}$, to the systems $B_{1}^{n}$.

(ii) Decode $\hat{m}_{1}$ by applying a second POVM $\left\{\Lambda_{m_{1} \mid \hat{m}_{0}}^{1}\right\}_{m_{1} \in\left[1: 2^{\left.n R_{1}\right]}\right.}$, to the systems $B_{1}^{n}$.

(iii) Choose $g$ to be the corresponding bin index such that $\hat{m}_{0} \in \mathscr{B}(g)$.

(iv) Send the conference message $g$ to Bob 2 .

where the POVMs $\left\{\Lambda_{m_{0}}^{0}\right\}$ and $\left\{\Lambda_{m_{1} \mid \hat{m}_{0}}^{1}\right\}$ will be specified later.

Bob 2 receives the systems $B_{2}^{n}$ and the conference message $g$ and decodes by applying a POVM $\left\{\Gamma_{m_{0}}\right\}_{m_{0} \in \mathscr{B}(g)}$, which will also be specified later, to the systems $B_{2}^{n}$.

Analysis of Probability of Error: Assume without loss of generality that Alice sends $\left(m_{0}, m_{1}\right)$. Denote the decoding measurement outcomes by $\widehat{M}_{0}, \widehat{M}_{1}, G$, and $\widetilde{M}_{0}$. Consider the following events,

$$
\begin{aligned}
& \mathscr{E}_{1}=\left\{\left(X_{0}^{n}\left(m_{0}\right), X_{1}^{n}\left(m_{0}, m_{1}\right)\right) \notin \mathscr{A}^{\delta / 2}\left(p_{X_{0}, X_{1}}\right)\right\} \\
& \mathscr{E}_{2}=\left\{\widehat{M}_{0} \neq m_{0}\right\} \\
& \mathscr{E}_{3}=\left\{\widehat{M}_{0}=m_{0}, \widehat{M}_{1} \neq m_{1}\right\} \\
& \mathscr{E}_{4}=\left\{\widetilde{M}_{0} \neq m_{0}\right\}
\end{aligned}
$$

By the union of events bound, the probability of error is bounded by

$$
P_{e \mid m_{0}, m_{1}}^{(n)}(\mathscr{F}, \Lambda, \Gamma) \leq \operatorname{Pr}\left(\mathscr{E}_{1}\right)+\operatorname{Pr}\left(\mathscr{E}_{2} \mid \mathscr{E}_{1}^{c}\right)+\operatorname{Pr}\left(\mathscr{E}_{3} \mid \mathscr{E}_{1}^{c} \cap \mathscr{E}_{2}^{c}\right)+\operatorname{Pr}\left(\mathscr{E}_{4} \mid \mathscr{E}_{1}^{c} \cap \mathscr{E}_{2}^{c} \cap \mathscr{E}_{3}^{c}\right)
$$

The first term tends to zero as $n \rightarrow \infty$ by the law of large numbers. To bound the second term, we use the quantum packing lemma. Given $\mathscr{E}_{1}^{c}$, we have $\left(X_{0}^{n}\left(m_{0}\right), X_{1}^{n}\left(m_{0}, m_{1}\right)\right) \in \mathscr{A}^{\delta / 2}\left(p_{X_{0}, X_{1}}\right)$. Now, 
Quantum Broadcast Channels with Cooperating Decoders

observe that

$$
\begin{aligned}
\Pi^{\delta}\left(\rho_{B_{1}}\right) \rho_{B_{1}^{n}} \Pi^{\delta}\left(\rho_{B_{1}}\right) & \preceq 2^{-n\left(H\left(B_{1}\right)_{\rho}-\varepsilon_{2}(\delta)\right)} \Pi^{\delta}\left(\rho_{B_{1}}\right) \\
\operatorname{Tr}\left[\Pi^{\delta}\left(\rho_{B_{1}} \mid x_{0}^{n}, x_{1}^{n}\right) \rho_{B_{1}{ }^{n}}^{x_{0}^{n}, x_{1}^{n}}\right] & \geq 1-\varepsilon_{2}(\delta) \\
\operatorname{Tr}\left[\Pi^{\delta}\left(\rho_{B_{1}} \mid x_{0}^{n}, x_{1}^{n}\right)\right] & \leq 2^{n\left(H\left(B_{1} \mid X_{0}, X_{1}\right)_{\rho}+\varepsilon_{2}(\delta)\right)} \\
\operatorname{Tr}\left[\Pi^{\delta}\left(\rho_{B_{1}}\right) \rho_{B_{1}{ }^{n}}^{x_{0}^{n}, x^{n}}\right] & \geq 1-\varepsilon_{2}(\delta)
\end{aligned}
$$

for all $\left(x_{0}^{n}, x_{1}^{n}\right) \in \mathscr{A}^{\delta / 2}\left(p_{X_{0}, X_{1}}\right)$, by (B4), (B6), (B8), and (B9), respectively. By the quantum packing lemma ${ }^{74}$ (see Ref. 119, Lem. 3), there exists a POVM $\Lambda_{\hat{m}_{0}}^{0}$ such that

$$
\operatorname{Tr}\left(\Lambda_{m_{0}}^{0} \rho_{B_{1}^{n}}^{x_{0}^{n}\left(m_{0}\right), x_{1}^{n}\left(m_{0}, m_{1}\right)}\right) \geq 1-2^{-n\left[H\left(B_{1}\right)_{\rho}-H\left(B_{1} \mid X_{0} X_{1}\right)_{\rho}-\left(R_{0}+R_{1}\right)-\varepsilon_{3}(\delta)\right]}
$$

for all $m_{0} \in\left[1: 2^{n R_{0}}\right]$. Hence, $\operatorname{Pr}\left(\mathscr{E}_{2} \mid \mathscr{E}_{1}^{c}\right) \leq 2^{-n\left(I\left(X_{0}, X_{1} ; B_{1}\right)_{\rho}-\left(R_{0}+R_{1}\right)-\varepsilon_{3}(\delta)\right)}$, which tends to zero as $n \rightarrow \infty$, provided that

$$
R_{0}+R_{1}<I\left(X_{0}, X_{1} ; B_{1}\right)_{\rho}-\varepsilon_{3}(\delta)
$$

Moving to the third term in the RHS of (B16), suppose that $\mathscr{E}_{2}^{c}$ occurred, namely the decoder measured the correct $M_{0}$. Denote the state of the systems $B_{1}^{n}$ after this measurement by $\rho_{B_{1}^{n}}^{\prime}$. Then, as in Refs. 119 and 120, we observe that due to the packing lemma inequality (B21), the gentle measurement lemma ${ }^{121,122}$ implies that the post-measurement state is close to the original state in the sense that

$$
\frac{1}{2}\left\|\rho_{B_{1}^{n}}^{\prime}-\rho_{B_{1}^{n}}\right\|_{1} \leq 2^{-n \frac{1}{2}\left(I\left(X_{0}, X_{1} ; B_{1}\right)_{\rho}-\left(R_{0}+R_{1}\right)-\varepsilon_{4}(\delta)\right)} \leq \varepsilon_{5}(\delta)
$$

for sufficiently large $n$ and rates as in (B22). Therefore, the distribution of measurement outcomes when $\rho_{B_{1}^{n}}^{\prime}$ is measured is roughly the same as if the POVM $\Lambda_{\hat{m}_{0}}^{0}$ was never performed. To be precise, the difference between the probability of a measurement outcome $\hat{m}_{1}$ when $\rho_{B_{1}^{n}}^{\prime}$ is measured and the probability when $\rho_{B_{1}^{n}}$ is measured is bounded by $\varepsilon_{5}(\boldsymbol{\delta})$ in absolute value (see Ref. 36, Lem. 9.11). Furthermore,

$$
\begin{aligned}
\operatorname{Tr}\left[\Pi^{\delta}\left(\rho_{B_{1}} \mid x_{0}^{n}, x_{1}^{n}\right) \rho_{B_{1}{ }^{n}}^{x_{0}^{n}, x^{n}}\right] & \geq 1-\varepsilon_{6}(\delta) \\
\Pi^{\delta}\left(\rho_{B} \mid x_{0}^{n}\right) \rho_{B_{1}{ }^{n}}^{x^{n}, x_{1}^{n}} \Pi^{\delta}\left(\rho_{B_{1}} \mid x_{0}^{n}\right) & \preceq 2^{-n\left(H\left(B_{1} \mid X_{0}\right)_{\rho}-\varepsilon_{6}(\delta)\right)} \Pi^{\delta}\left(\rho_{B_{1}} \mid x_{0}^{n}\right) \\
\operatorname{Tr}\left[\Pi^{\delta}\left(\rho_{B_{1}} \mid x_{0}^{n}, x_{1}^{n}\right)\right] & \leq 2^{n\left(H\left(B_{1} \mid X_{0}, X_{1}\right)_{\rho}+\varepsilon_{6}(\delta)\right)} \\
\operatorname{Tr}\left[\Pi^{\delta}\left(\rho_{B_{1}} \mid x_{0}^{n}\right) \rho_{B_{1}{ }^{n}}^{x_{0}^{n}, x^{n}}\right] & \geq 1-\varepsilon_{6}(\delta)
\end{aligned}
$$


for all $\left(x_{0}^{n}, x_{1}^{n}\right) \in \mathscr{A}^{\delta / 2}\left(p_{X_{0}} p_{X_{1} \mid X_{0}}\right)$, by (B6), (B7), (B8), and (B9), respectively. Therefore, we have by the quantum packing lemma that there exists a POVM $\Lambda_{\hat{m}_{1} \mid m_{0}}^{1}$ such that $\operatorname{Pr}\left(\mathscr{E}_{3} \mid \mathscr{E}_{1} c \cap \mathscr{E}_{2} c\right) \leq$ $2^{-n\left(I\left(X_{1} ; B_{1} \mid X_{0}\right)_{\rho}-R_{1}-\varepsilon_{7}(\delta)\right)}$, which tends to zero as $n \rightarrow \infty$, provided that

$$
R_{1}<I\left(X_{1} ; B_{1} \mid X_{0}\right)_{\rho}-\varepsilon_{7}(\delta)
$$

It remains to consider erroneous decoding by Bob 2. Suppose that $\mathscr{E}_{3} c$ occurred, namely Bob 1 measured the correct $m_{0}$, and thus sent the correct bin index $G$ such that $m_{0} \in \mathscr{B}(G)$. Recall that the size of each bin is $|\mathscr{B}(G)|=2^{n\left(R_{0}-\mathrm{C}_{12}\right)}$ (see (B10)). Then, observe that

$$
\begin{aligned}
\Pi^{\delta}\left(\rho_{B_{2}}\right) \rho_{B_{2}} \Pi^{\delta}\left(\rho_{B_{2}}\right) & \preceq 2^{-n\left(H\left(B_{2}\right)_{\rho}-\varepsilon_{2}(\delta)\right)} \Pi^{\delta}\left(\rho_{B_{1}}\right) \\
\operatorname{Tr}\left[\Pi^{\delta}\left(\rho_{B_{2}} \mid x_{0}^{n}\right) \sigma_{B_{2}{ }^{n}}^{x^{n}}\right] & \geq 1-\varepsilon_{2}(\delta) \\
\operatorname{Tr}\left[\Pi^{\delta}\left(\rho_{B_{2}} \mid x_{0}^{n}\right)\right] & \leq 2^{n\left(H\left(B_{2} \mid X_{0}\right)_{\rho}+\varepsilon_{2}(\delta)\right)} \\
\operatorname{Tr}\left[\Pi^{\delta}\left(\rho_{B_{2}}\right) \rho_{B_{2}{ }^{n}}^{x^{n}}\right] & \geq 1-\varepsilon_{2}(\delta)
\end{aligned}
$$

for all $x_{0}^{n} \in \mathscr{A}^{\delta}\left(p_{X_{0}, X_{1}}\right)$, by (B4), (B6), (B8), and (B9), respectively. Hence, by the quantum packing lemma (Ref. 119, Lem. 3), there exists a POVM $\Gamma_{\widetilde{m}_{0}}$ such that $\operatorname{Pr}\left(\mathscr{E}_{4} \mid \mathscr{E}_{1}^{c} \cap \mathscr{E}_{2}^{c} \cap \mathscr{E}_{3} c\right) \leq$ $2^{-n\left(I\left(X_{0} ; B_{2}\right)_{\rho}-\left(R_{0}-C_{12}\right)-\varepsilon_{8}(\delta)\right)}$, which tends to zero as $n \rightarrow \infty$, provided that

$$
R_{0}<I\left(X_{0} ; B_{2}\right)_{\rho}+C_{12}-\varepsilon_{8}(\delta)
$$

To show that rate pairs in $\frac{1}{\kappa} \mathscr{R}_{\mathrm{Cl}}\left(\mathscr{N}^{\otimes \kappa}\right)$ are achievable as well, one may employ the coding scheme above for the product broadcast channel $\mathscr{N}^{\otimes \kappa}$, where $\kappa$ is arbitrarily large. This completes the proof of the direct part.

\section{Converse Proof}

Consider the converse part for the regularized capacity formula. Suppose that Alice chooses $m_{0}$ and $m_{1}$ uniformly at random, and prepares an input state $\rho_{A^{n}}^{m_{0}, m_{1}}$. After Alice sends the systems $A^{n}$ through the channel, the output state is $\rho_{B_{1}^{n} B_{2}^{n}}=\frac{1}{2^{n\left(R_{0}+R_{1}\right)}} \sum_{m_{0}, m_{1}}^{2^{n R_{0}}} \sum_{m_{1}=1}^{2^{n R_{1}}} \mathscr{N}_{A^{n} \rightarrow B_{1}^{n} B_{2}^{n}}\left(\rho_{A^{n}}^{m_{0}, m_{1}}\right)$. Then, Bob 1 performs a decoding POVM $\Lambda_{B_{1}^{n}}^{m_{0}, m_{1}, g}$, sends $g$ to Bob 2 using conferencing, and Bob 2 chooses a POVM $\Gamma_{B_{2}^{n} \mid g}^{m_{0}}$ accordingly. Consider a sequence of codes $\left(\mathscr{F}_{n}, \Lambda_{n}, \Gamma_{n}\right)$ such that the average probability of error tends to zero, hence the error probabilities $\operatorname{Pr}\left(\widehat{M}_{0} \neq M_{0}\right)$, $\operatorname{Pr}\left(\left(\widehat{M}_{0}, \widehat{M}_{1}\right) \neq\left(M_{0}, M_{1}\right)\right), \operatorname{Pr}\left(\widehat{M}_{1} \neq M_{1} \mid M_{0}\right)$ are bounded by some $\alpha_{n}$ which tends to zero as 
Quantum Broadcast Channels with Cooperating Decoders

$n \rightarrow \infty$. By Fano's inequality ${ }^{123}$, it follows that

$$
\begin{array}{r}
H\left(M_{0} \mid \widetilde{M}_{0}\right) \leq n \varepsilon_{n} \\
H\left(M_{0}, M_{1} \mid \widehat{M}_{0}, \widehat{M}_{1}\right) \leq n \varepsilon_{n}^{\prime} \\
H\left(M_{1} \mid \widehat{M}_{1}, M_{0}\right) \leq n \varepsilon_{n}^{\prime \prime}
\end{array}
$$

where $\varepsilon_{n}, \varepsilon_{n}^{\prime}, \varepsilon_{n}^{\prime \prime}$ tend to zero as $n \rightarrow \infty$. Hence,

$$
\begin{aligned}
n R_{0} & =H\left(M_{0}\right)=I\left(M_{0} ; \widetilde{M}_{0}\right)_{\rho}+H\left(M_{0} \mid \widetilde{M}_{0}\right) \leq I\left(M_{0} ; \widetilde{M}_{0}\right)_{\rho}+n \varepsilon_{n} \\
& \leq I\left(M_{0} ; B_{2}^{n} G\right)_{\rho}+n \varepsilon_{n}=I\left(M_{0} ; B_{2}^{n}\right)_{\rho}+I\left(M_{0} ; G \mid B_{2}^{n}\right)_{\rho}+n \varepsilon_{n} \\
& \leq I\left(M_{0} ; B_{2}^{n}\right)_{\rho}+n C_{12}+n \varepsilon_{n}
\end{aligned}
$$

where the second inequality follows from the Holevo bound (see Ref. 33, Theo. 12.1), and the last inequality holds as $I\left(M_{0} ; G \mid B_{2}^{n}\right)_{\rho} \leq H(G) \leq n C_{12}$ because $G$ is a classical message in $\left[1: 2^{n C_{12}}\right]$. Similarly,

$$
n\left(R_{0}+R_{1}\right) \leq I\left(M_{0}, M_{1} ; B_{1}^{n}\right)_{\rho}+n \varepsilon_{n}^{\prime}
$$

Furthermore, since $M_{0}$ and $M_{1}$ are statistically independent, we can also write

$$
n R_{1}=H\left(M_{1} \mid M_{0}\right) \leq I\left(M_{1} ; \widehat{M}_{1} \mid M_{0}\right)_{\rho}+n \varepsilon_{n}^{\prime \prime} \leq I\left(M_{1} ; B_{1}^{n} \mid M_{0}\right)_{\rho}+n \varepsilon_{n}^{\prime \prime}
$$

We deduce that $R_{0} \leq I\left(X_{0}^{n} ; B_{2}^{n}\right)_{\rho}+\mathrm{C}_{12}+\varepsilon_{n}, R_{0}+R_{1} \leq I\left(X_{0}^{n}, X_{1}^{n} ; B_{1}^{n}\right)_{\rho}+\varepsilon_{n}^{\prime}$, and $R_{1} \leq I\left(X_{1}^{n} ; B_{1}^{n} \mid X_{0}^{n}\right)_{\rho}+$ $\varepsilon_{n}^{\prime \prime}$ with $X_{k}^{n}=f_{k}\left(M_{k}\right)$ where $f_{k}$ are arbitrary one-to-one maps from $\left[1: 2^{n R_{k}}\right]$ to $\mathscr{X}_{k}^{n}$, for $k=0,1$. This completes the converse proof for the regualarized characterization.

For a Hadamard broadcast channel, where Bob 1 receives a classical output $Y_{1}^{n}$, define

$$
X_{0, i}=\left(M_{0}, Y_{1}^{i-1}\right), X_{1, i}=\left(M_{1}, Y_{1}^{i-1}\right)
$$

Applying the chain rule to (B37),

$$
n R_{0} \leq \sum_{i=1}^{n} I\left(M_{0} ; B_{2, i} \mid B_{2}^{i-1}\right)_{\rho}+n C_{12}+n \varepsilon_{n} \leq \sum_{i=1}^{n} I\left(M_{0} B_{2}^{i-1} ; B_{2, i}\right)_{\rho}+n C_{12}+n \varepsilon_{n} .
$$

Since the marginal of Bob 2 is degraded with respect to that of Bob 1 , namely $\mathscr{N}_{A \rightarrow B_{2}}^{\mathrm{H}}=$ $\mathscr{P}_{Y_{1} \rightarrow B_{2}} \circ \mathscr{N}_{A \rightarrow Y_{1}}^{\mathrm{H}}$, the data processing inequality for the quantum mutual information implies that $I\left(M_{0} B_{2}^{i-1} ; B_{2, i}\right)_{\rho} \leq I\left(M_{0} Y_{1}^{i-1} ; B_{2, i}\right)_{\rho}=I\left(X_{0, i} ; B_{2, i}\right)_{\rho}$, hence

$$
R_{0}-\varepsilon_{n} \leq \frac{1}{n} \sum_{i=1}^{n} I\left(X_{0, i} ; B_{2, i}\right)_{\rho}+C_{12}=I\left(X_{0, K} ; B_{2, K} \mid K\right)_{\rho}+C_{12}
$$


where $K$ is a classical random variable with uniform distribution over $[1: n]$, independent of $M_{0}$, $M_{1}$, and $G$. Defining $\rho_{K A}=\frac{1}{n} \sum_{i=1}^{n}|i\rangle\langle i| \otimes \rho_{A_{i}}, \rho_{K B_{1} B_{2}}=\mathscr{N}_{A \rightarrow B_{1} B_{2}}\left(\rho_{K A}\right)$, and

$$
X_{0} \equiv\left(X_{0, K}, K\right), X_{1} \equiv\left(X_{1, K}, K\right)
$$

we obtain

$$
R_{0} \leq I\left(X_{0} ; B_{2}\right)_{\rho}+\mathrm{C}_{12}+\varepsilon_{n}
$$

and by similar considerations,

$$
\begin{aligned}
R_{0}+R_{1} & \leq I\left(X_{0} X_{1} ; B_{1}\right)+\varepsilon_{n} \\
R_{1} & \leq I\left(X_{1} ; B_{1} \mid X_{0}\right)+\varepsilon_{n}
\end{aligned}
$$

This completes the proof of Theorem 2 .

\section{REFERENCES}

${ }^{1}$ L. Jiang, J. M. Taylor, K. Nemoto, W. J. Munro, R. Van Meter, and M. D. Lukin, “Quantum repeater with encoding," Phys. Rev. A 79, 032325 (2009).

${ }^{2}$ B. K. Behera, S. Seth, A. Das, and P. K. Panigrahi, "Demonstration of entanglement purification and swapping protocol to design quantum repeater in ibm quantum computer," Quantum Information Processing 18, 108 (2019).

${ }^{3}$ R. Bassoli, R. Ferrara, S. Saeedinaeeni, C. Deppe, H. Boche, F. H. P. Fitzek, and G. Jansen, Quantum Communication Networks (Springer Nature (unpublished), 2020).

${ }^{4}$ H. J. Briegel, W. Dür, J. I. Cirac, and P. Zoller, “Quantum repeaters: the role of imperfect local operations in quantum communication,” Phys. Rev. Lett. 81, 5932 (1998).

${ }^{5}$ P. van Loock, W. Alt, C. Becher, O. Benson, H. Boche, C. Deppe, J. Eschner, S. Höfling, D. Meschede, and P. Michler, "Extending quantum links: Modules for fiber-and memory-based quantum repeaters,” Adv. Quantum Technol. , 1900141 (2020).

${ }^{6}$ C. Gies, M. Florian, A. Steinhoff, and F. Jahnke, Quantum Dots for Quantum Information Technologies (Springer International Publishing, New York, 2017).

${ }^{7}$ V. Krutyanskiy, M. Meraner, J. Schupp, V. Krcmarsky, H. Hainzer, and B. P. Lanyon, "Lightmatter entanglement over $50 \mathrm{~km}$ of optical fibre," npj Quantum Information 5, 1-5 (2019). 
${ }^{8}$ C. Nguyen, D. Sukachev, M. Bhaskar, B. Machielse, D. Levonian, E. Knall, P. Stroganov, C. Chia, M. Burek, and R. Riedinger, "An integrated nanophotonic quantum register based on silicon-vacancy spins in diamond," Phys. Rev. B 100, 165428 (2019).

${ }^{9}$ F. Rozpędek, R. Yehia, K. Goodenough, M. Ruf, P. C. Humphreys, R. Hanson, S. Wehner, and D. Elkouss, "Near-term quantum-repeater experiments with nitrogen-vacancy centers: Overcoming the limitations of direct transmission,” Phys. Rev. A 99, 052330 (2019).

${ }^{10}$ J. P. Dowling and G. J. Milburn, “Quantum technology: the second quantum revolution,” Philos. Trans. Royal Soc. London. Series A: Math., Phys. and Eng. Sciences 361, 1655-1674 (2003).

${ }^{11}$ P. Zoller, T. Beth, D. Binosi, R. Blatt, H. Briegel, D. Bruss, T. Calarco, J. I. Cirac, D. Deutsch, J. Eisert, A. Ekert, et al., "Quantum information processing and communication," Euro. Phys. J. D-Atom. Molec. Optic. Plasma Phys. 36, 203-228 (2005).

${ }^{12}$ P. Jouguet, S. Kunz-Jacques, A. Leverrier, P. Grangier, and E. Diamanti, "Experimental demonstration of long-distance continuous-variable quantum key distribution," Nature Photonics 7, 378 (2013).

${ }^{13}$ F. E. Becerra, J. Fan, and A. Migdall, "Photon number resolution enables quantum receiver for realistic coherent optical communications," Nature Photonics 9, 48 (2015).

${ }^{14}$ J. Yin, Y. Cao, Y. H. Li, S. K. Liao, L. Zhang, J. G. Ren, W. Q. Cai, W. Y. Liu, B. Li, H. Dai, G. B. Li, Q. M. Lu, Y. H. Gong, Y. Xu, S. L. Li, F. Z. Li, Y. Y. Yin, Z. Q. Jiang, M. Li, J. J. Jia, G. Ren, D. He, Y. L. Zhou, X. X. Zhang, N. Wang, X. Chang, Z. C. Zhu, N. L. Liu, Y. A. Chen, C. Y. Lu, R. Shu, C. Z. Peng, J. Y. Wang, and J. W. Pan, "Satellite-based entanglement distribution over 1200 kilometers," Science 356, 1140-1144 (2017), https://science.sciencemag.org/content/356/6343/1140.full.pdf.

${ }^{15}$ W. Zhang, D. S. Ding, Y. B. Sheng, L. Zhou, B. S. Shi, and G. C. Guo, "Quantum secure direct communication with quantum memory,” Phys. Rev. Lett. 118, 220501 (2017).

${ }^{16}$ Y. Liu, Z. W. Yu, W. Zhang, J. Y. Guan, J. P. Chen, C. Zhang, X. L. Hu, H. Li, C. Jiang, and J. Lin, "Experimental twin-field quantum key distribution through sending or not sending," Phys. Rev. Lett. 123, 100505 (2019).

${ }^{17}$ L. Petit, H. G. J. Eenink, M. Russ, W. I. L. Lawrie, N. W. Hendrickx, S. G. J. Philips, J. S. Clarke, L. M. K. Vandersypen, and M. Veldhorst, "Universal quantum logic in hot silicon qubits," Nature 580, 355-359 (2020).

${ }^{18}$ L. Gyongyosi, S. Imre, and H. V. Nguyen, “A survey on quantum channel capacities," IEEE Commun. Surveys Tutorials 20, 1149-1205 (2018). 
Quantum Broadcast Channels with Cooperating Decoders

${ }^{19}$ G. Smith and J. Yard, "Quantum communication with zero-capacity channels," Science 321, $1812-1815$ (2008).

${ }^{20}$ H. Boche and R. F. Schaefer, "Capacity results and super-activation for wiretap channels with active wiretappers,” IEEE Trans. Inf. Forens. Secur. 8, 1482-1496 (2013).

${ }^{21}$ H. Boche, R. F. Schaefer, and H. V. Poor, "Identification capacity of channels with feedback: Discontinuity behavior, super-activation, and turing computability," IEEE Transactions on Information Theory 66, 6184-6199 (2020).

${ }^{22}$ A. S. Holevo, “The capacity of the quantum channel with general signal states," IEEE Trans. Inf. Theory 44, 269-273 (1998).

${ }^{23}$ B. Schumacher and M. D. Westmoreland, "Sending classical information via noisy quantum channels," Phys. Rev. A 56, 131 (1997).

${ }^{24}$ A. S. Holevo, Quantum systems, channels, information: a mathematical introduction, Vol. 16 (Walter de Gruyter, 2012).

${ }^{25}$ H. Barnum, M. A. Nielsen, and B. Schumacher, "Information transmission through a noisy quantum channel,” Phys. Rev. A 57, 4153 (1998).

${ }^{26}$ S. Lloyd, "Capacity of the noisy quantum channel," Phys. Rev. A 55, 1613 (1997).

${ }^{27}$ P. W. Shor, "The quantum channel capacity and coherent information," in Lecture notes, MSRI Workshop Quant. Comput. (2002).

${ }^{28}$ I. Devetak, “The private classical capacity and quantum capacity of a quantum channel," IEEE Trans. Inf. Theory 51, 44-55 (2005).

${ }^{29}$ I. Devetak and P. W. Shor, “The capacity of a quantum channel for simultaneous transmission of classical and quantum information," Commun. in Math. Phys. 256, 287-303 (2005).

${ }^{30} \mathrm{~S}$. Watanabe, "Private and quantum capacities of more capable and less noisy quantum channels," Phys. Rev. A 85, 012326 (2012).

${ }^{31}$ I. Bjelaković, H. Boche, and J. Nötzel, "Entanglement transmission and generation under channel uncertainty: Universal quantum channel coding," Commun. in Math. Phys. 292, 55-97 (2009).

${ }^{32}$ M. M. Wilde and M. Hsieh, "Entanglement generation with a quantum channel and a shared state," in Proc. IEEE Int. Symp. Inf. Theory (ISIT'2010) (Austin, TX, USA, 2010) pp. 27132717.

${ }^{33}$ M. A. Nielsen and I. Chuang, "Quantum computation and quantum information,” (2002).

${ }^{34}$ H. Boche, G. Janßen, and S. Kaltenstadler, "Entanglement-assisted classical capacities of 
Quantum Broadcast Channels with Cooperating Decoders

compound and arbitrarily varying quantum channels," Quantum Information Processing 16, 88 (2017).

${ }^{35}$ U. Pereg, C. Deppe, and H. Boche, “Quantum channel state masking,” IEEE Trans. Inf. Theory 67, 2245-2268 (2021).

${ }^{36}$ M. M. Wilde, Quantum information theory, 2nd ed. (Cambridge University Press, 2017).

${ }^{37}$ E. Chitambar and G. Gour, "Quantum resource theories," Rev. Modern Phys. 91, 025001 (2019).

${ }^{38}$ C. H. Bennett and S. J. Wiesner, "Communication via one-and two-particle operators on einstein-podolsky-rosen states," Phys. Rev. Lett. 69, 2881 (1992).

${ }^{39}$ C. H. Bennett, P. W. Shor, J. A. Smolin, and A. V. Thapliyal, "Entanglement-assisted classical capacity of noisy quantum channels," Phys. Rev. Lett. 83, 3081 (1999).

${ }^{40}$ C. H. Bennett, P. W. Shor, J. A. Smolin, and A. V. Thapliyal, "Entanglement-assisted capacity of a quantum channel and the reverse shannon theorem,” IEEE Trans. Inf. Theory 48, 26372655 (2002).

${ }^{41}$ B. Swingle, "Mutual information and the structure of entanglement in quantum field theory," arXiv:1010.4038 (2010).

${ }^{42}$ Q. Pan and J. Jing, "Degradation of nonmaximal entanglement of scalar and dirac fields in noninertial frames," Phys. Rev. A 77, 024302 (2008).

${ }^{43}$ H. Casini, M. Huerta, R. C. Myers, and A. Yale, "Mutual information and the f-theorem," J. High Energy Phys. 2015, 3 (2015).

${ }^{44}$ C. A. Agón and T. Faulkner, “Quantum corrections to holographic mutual information,” J. High Energy Phys. 2016, 118 (2016).

${ }^{45}$ F. Leditzky, M. A. Alhejji, J. Levin, and G. Smith, "Playing games with multiple access channels," Nature communications 11, 1-5 (2020).

${ }^{46}$ G. Brassard, A. Broadbent, and A. Tapp, “Quantum pseudo-telepathy,” Foundations of Physics 35, 1877-1907 (2005).

${ }^{47}$ J. Nötzel, “Entanglement-enabled communication,” arXiv:1910.03796 (2019).

${ }^{48}$ Y. Quek and P. W. Shor, "Quantum and superquantum enhancements to two-sender, tworeceiver channels,” Phys. Rev. A 95, 052329 (2017).

${ }^{49}$ J. F. Clauser, M. A. Horne, A. Shimony, and R. A. Holt, "Proposed experiment to test local hidden-variable theories," Physical review letters 23, 880 (1969).

${ }^{50}$ A. Pappa, A. Chailloux, S. Wehner, E. Diamanti, and I. Kerenidis, "Multipartite entanglement 
verification resistant against dishonest parties,” Phys. Rev. Lett. 108, 260502 (2012).

${ }^{51}$ U. Vazirani and T. Vidick, "Fully device-independent quantum key distribution," Phys. Rev. Lett. 113, 140501 (2014).

${ }^{52}$ Z. A. Jia, L. Wei, Y. C. Wu, and G. C. Guo, "Quantum advantages of communication complexity from bell nonlocality," arXiv:2004.05098 (2020).

${ }^{53}$ Z. Ji, A. Natarajan, T. Vidick, J. Wright, and H. Yuen, “Mip*= re," arXiv:2001.04383 (2020).

${ }^{54}$ C. H. Bennett, G. Brassard, C. Crépeau, R. Jozsa, A. Peres, and W. K. Wootters, “Teleporting an unknown quantum state via dual classical and einstein-podolsky-rosen channels," Physical review letters 70, 1895 (1993).

${ }^{55}$ J. Yard, P. Hayden, and I. Devetak, “Quantum broadcast channels,” IEEE Trans. Inf. Theory 57, 7147-7162 (2011).

${ }^{56}$ I. Savov and M. M. Wilde, "Classical codes for quantum broadcast channels," IEEE Trans. Inf. Theory 61, 7017-7028 (2015).

${ }^{57}$ J. Radhakrishnan, P. Sen, and N. Warsi, "One-shot marton inner bound for classical-quantum broadcast channel," IEEE Trans. Inf. Theory 62, 2836-2848 (2016).

${ }^{58}$ Q. Wang, S. Das, and M. M. Wilde, "Hadamard quantum broadcast channels," Quantum Inform. Process. 16, 248 (2017).

${ }^{59}$ F. Dupuis, P. Hayden, and K. Li, “A father protocol for quantum broadcast channels,” IEEE Trans. Inf. Theory 56, 2946-2956 (2010).

${ }^{60} \mathrm{~F}$. Dupuis, The decoupling approach to quantum information theory, $\mathrm{Ph} . \mathrm{D}$. thesis, Université de Montréal (2010).

${ }^{61} \mathrm{C}$. Hirche and C. Morgan, "An improved rate region for the classical-quantum broadcast channel," in Proc. IEEE Int. Symp. Inf. Theory (ISIT'2015) (Hong Kong, China, 2015) pp. 27822786.

${ }^{62}$ K. P. Seshadreesan, M. Takeoka, and M. M. Wilde, "Bounds on entanglement distillation and secret key agreement for quantum broadcast channels,” IEEE Trans. Inf. Theory 62, 2849-2866 (2016).

${ }^{63}$ S. Bäuml and K. Azuma, "Fundamental limitation on quantum broadcast networks," Quantum Science and Tech. 2, 024004 (2017).

${ }^{64}$ T. Heinosaari and T. Miyadera, "Incompatibility of quantum channels," J. Phys. A: Mat. and Theo. 50, 135302 (2017). 
Quantum Broadcast Channels with Cooperating Decoders

${ }^{65}$ H. Boche, M. Cai, and C. Deppe, "The broadcast classical-quantum capacity region of a twophase bidirectional relaying channel," Quantum Information Processing 14, 3879-3897 (2015).

${ }^{66} \mathrm{C}$. Hirche, Polar codes in quantum information theory, Ph.D. thesis, Leibniz Universität Hannover (2015).

${ }^{67}$ W. Xie, X. Wang, and R. Duan, “Converse bounds for classical communication over quantum broadcast channels and quantum multi-access channels," in Proc. IEEE Int. Symp. Inf. Theory (ISIT'2018) (2018) pp. 2341-2345.

${ }^{68}$ G. De Palma, "New lower bounds to the output entropy of multi-mode quantum gaussian channels,” IEEE Trans. Inf. Theory 65, 5959-5968 (2019).

${ }^{69}$ A. Anshu, R. Jain, and N. A. Warsi, "Building blocks for communication over noisy quantum networks," IEEE Trans. Inf. Theory 65, 1287-1306 (2019).

${ }^{70}$ H. C. Cheng, N. Datta, and C. Rouzé, "Strong converse for classical-quantum degraded broadcast channels," arXiv:1905.00874 (2019).

${ }^{71}$ A. Winter, “The capacity of the quantum multiple-access channel,” IEEE Trans. Inf. Theory 47, 3059-3065 (2001).

${ }^{72}$ G. V. Klimovitch, "On the classical capacity of a quantum multiple-access channel," in Proc. IEEE Int. Symp. Inf. Theory (ISIT’2001) (Washington, DC, USA, 2001) pp. 278-.

${ }^{73} \mathrm{~J}$. Yard, Simultaneous classical-quantum capacities of quantum multiple access channels, Ph.d. dissertation, Stanford University (2005).

${ }^{74}$ M. Hsieh, I. Devetak, and A. Winter, "Entanglement-assisted capacity of quantum multipleaccess channels," IEEE Trans. Inf. Theory 54, 3078-3090 (2008).

${ }^{75}$ J. Yard, P. Hayden, and I. Devetak, "Capacity theorems for quantum multiple-access channels: classical-quantum and quantum-quantum capacity regions,” IEEE Trans. Inf. Theory 54, 30913113 (2008).

${ }^{76}$ L. Czekaj and P. Horodecki, "Purely quantum superadditivity of classical capacities of quantum multiple access channels,” Phys. Rev. Lett. 102, 110505 (2009).

${ }^{77}$ H. Boche and J. Noetzel, “The classical-quantum multiple access channel with conferencing encoders and with common messages," Quantum Info. Proc. 13, 2595-2617 (2014).

${ }^{78} \mathrm{~S}$. Diadamo and H. Boche, "The simultaneous identification capacity of the classical-quantum multiple access channel with stochastic encoders for transmission," arXiv:1903.03395 (2019).

${ }^{79}$ M. Koashi and A. Winter, "Monogamy of quantum entanglement and other correlations," Phys. 
Quantum Broadcast Channels with Cooperating Decoders

Rev. A 69, 022309 (2004).

${ }^{80}$ S. Guha and J. H. Shapiro, "Classical information capacity of the bosonic broadcast channel," in Proc. IEEE Int. Symp. Inf. Theory (ISIT’2007) (Nice, France, 2007) pp. 1896-1900.

${ }^{81}$ S. Guha, J. H. Shapiro, and B. I. Erkmen, "Classical capacity of bosonic broadcast communication and a minimum output entropy conjecture," Phys. Rev. A 76, 032303 (2007).

${ }^{82}$ G. De Palma, A. Mari, and V. Giovannetti, “A generalization of the entropy power inequality to bosonic quantum systems," Nature Photonics 8, 958 (2014).

${ }^{83}$ M. Takeoka, K. P. Seshadreesan, and M. M. Wilde, "Unconstrained distillation capacities of a pure-loss bosonic broadcast channel," in Proc. IEEE Int. Symp. Inf. Theory (ISIT'2016) (Barcelona, Spain, 2016) pp. 2484-2488.

${ }^{84}$ M. Takeoka, K. P. Seshadreesan, and M. M. Wilde, "Unconstrained capacities of quantum key distribution and entanglement distillation for pure-loss bosonic broadcast channels," Phys. Rev. Lett. 119, 150501 (2017).

${ }^{85}$ F. Salek, M. H. Hsieh, and J. R. Fonollosa, "Publicness, privacy and confidentiality in the single-serving quantum broadcast channel," arXiv:1903.04463 (2019).

${ }^{86}$ F. Salek, M. Hsieh, and J. R. Fonollosa, "Publicness, privacy and confidentiality in the singleserving quantum broadcast channel," in Proc. IEEE Int. Symp. Inf. Theory (ISIT'2019) (Paris, France, 2019) pp. 1712-1716.

${ }^{87}$ H. Aghaee and B. Akhbari, “Classical-quantum multiple access wiretap channel," in Int'l ISC Conf. Info. Secur. Crypt. (ISCISC'2019) (Mashhad, Iran, 2019).

${ }^{88}$ H. Boche, G. Janßen, and S. Saeedinaeeni, “Universal superposition codes: Capacity regions of compound quantum broadcast channel with confidential messages,” J. Math. Phys. 61, 042204 (2020).

${ }^{89}$ I. Savov, M. M. Wilde, and M. Vu, "Partial decode-forward for quantum relay channels," in Proc. IEEE Int. Symp. Inf. Theory (ISIT'2012) (Cambridge, MA, USA, 2012) pp. 731-735.

${ }^{90}$ I. Savov, Network information theory for classical-quantum channels, Ph.D. thesis, McGill University, Montreal (2012).

${ }^{91}$ D. Ding, H. Gharibyan, P. Hayden, and M. Walter, “A quantum multiparty packing lemma and the relay channel," IEEE Trans. Inf. Theory 66, 3500-3519 (2020).

${ }^{92}$ P. Hayden and C. King, "Correcting quantum channels by measuring the environment," arXiv:quant-ph/0409026 (2004).

${ }^{93}$ J. A. Smolin, F. Verstraete, and A. Winter, "Entanglement of assistance and multipartite state 
Quantum Broadcast Channels with Cooperating Decoders

distillation,” Phys. Rev. A 72, 052317 (2005).

${ }^{94}$ A. Winter, “On environment-assisted capacities of quantum channels," arXiv:quant-ph/0507045 (2005).

${ }^{95}$ R. Dabora and S. D. Servetto, "Broadcast channels with cooperating decoders," IEEE Transactions on Information Theory 52, 5438-5454 (2006).

${ }^{96}$ Y. Steinberg, "Instances of the relay-broadcast channel and cooperation strategies," in Proc. IEEE Int. Symp. Inf. Theory (ISIT’2015) (Hong Kong, China, 2015) pp. 2653-2657.

${ }^{97}$ Y. H. Kim, "Coding techniques for primitive relay channels," in Proc. Allerton Conf. Commun., Control, Computing (Monticello, Illinois, 2007) pp. 129-135.

${ }^{98}$ M. Berta, F. G. S. L. Brandão, M. Christandl, and S. Wehner, "Entanglement cost of quantum channels," IEEE Transactions on Information Theory 59, 6779-6795 (2013).

${ }^{99}$ L. Gyongyosi and S. Imre, "Private quantum coding for quantum relay networks," in Meeting Euro. Netw. Univ. Comp. Inf. Commun. Engin. (Springer, 2012) pp. 239-250.

${ }^{100}$ S. Jin-Jing, S. Rong-Hua, P. Xiao-Qi, G. Ying, Y. Liu-Yang, and L. Moon-Ho, "Lower bounds on the capacities of quantum relay channels," Commun. Theo. Phys. 58, 487 (2012).

${ }^{101}$ L. Gyongyosi and S. Imre, "Reliable quantum communication over a quantum relay channel," AIP Conf. Proc., 1633, 165-167 (2014).

${ }^{102}$ S. Pirandola, "Capacities of repeater-assisted quantum communications," arXiv: 1601.00966 (2016).

${ }^{103}$ M. Ghalaii and S. Pirandola, "Capacity-reaching quantum repeaters for quantum communications," arXiv:2007.06988 (2020).

${ }^{104}$ N. Jindal, S. Vishwanath, and A. Goldsmith, "On the duality of gaussian multiple-access and broadcast channels,” IEEE Trans. Inf. Theory 50, 768-783 (2004).

${ }^{105} \mathrm{P}$. Viswanath and D. N. C. Tse, "Sum capacity of the vector gaussian broadcast channel and uplink-downlink duality,” IEEE Trans. Inf. Theory 49, 1912-1921 (2003).

${ }^{106}$ H. Weingarten, Y. Steinberg, and S. S. Shamai, "The capacity region of the gaussian multipleinput multiple-output broadcast channel," IEEE Transactions on Information Theory 52, 39363964 (2006).

${ }^{107}$ C. H. Bennett, D. P. DiVincenzo, J. A. Smolin, and W. K. Wootters, "Mixed-state entanglement and quantum error correction,” Physical Review A 54, 3824 (1996).

${ }^{108}$ P. W. Shor, "Additivity of the classical capacity of entanglement-breaking quantum channels," J. Math. Phys. 43, 4334-4340 (2002). 
Quantum Broadcast Channels with Cooperating Decoders

${ }^{109}$ H. Boche, M. Cai, N. Cai, and C. Deppe, "Secrecy capacities of compound quantum wiretap channels and applications," Phys. Rev. A 89, 052320 (2014).

${ }^{110}$ E. C. van der Meulen, “Three-terminal communication channels,” Adv. Appl. Prob. 3, 120-154 (1971).

${ }^{111}$ R. Ahlswede, I. Bjelaković, H. Boche, and J. Nötzel, "Quantum capacity under adversarial quantum noise: arbitrarily varying quantum channels," Commun. in Math. Phys. 317, 103-156 (2013).

${ }^{112}$ R. Alicki and M. Fannes, "Continuity of quantum conditional information,” J. Phys. A: Math. General 37, L55-L57 (2004).

${ }^{113}$ A. Winter, "Tight uniform continuity bounds for quantum entropies: conditional entropy, relative entropy distance and energy constraints," Commun. in Math. Phys. 347, 291-313 (2016).

${ }^{114}$ E. Telatar, “Capacity of multi-antenna gaussian channels,” Euro. Trans. Telecomm. 10, 585-595 (1999).

${ }^{115}$ A. El Gamal and Y. Kim, Network Information Theory (Cambridge University Press, 2011).

${ }^{116}$ F. R. Farrokhi, K. J. R. Liu, and L. Tassiulas, "Transmit beamforming and power control in wireless networks with fading channels," IEEE J. Select. Areas Commun. 16, 1437-1450 (1998).

${ }^{117}$ H. Boche and M. Schubert, "A general duality theory for uplink and downlink beamforming," in Proc. IEEE 56th Vehic. Tech. Conf., Vol. 1 (IEEE, 2002) pp. 87-91.

${ }^{118}$ H. G. Eggleston, “Convexity,” J. London Math. Society 1, 183-186 (1966).

${ }^{119}$ U. Pereg, "Communication over quantum channels with parameter estimation," arXiv:2001.00836 (2020).

${ }^{120}$ U. Pereg, "Communication over quantum channels with parameter estimation," in Proc. IEEE Int. Symp. Inf. Theory (ISIT'2020) (2020).

${ }^{121}$ A. Winter, "Coding theorem and strong converse for quantum channels," IEEE Trans. Inf. Theory 45, 2481-2485 (1999).

${ }^{122}$ T. Ogawa and H. Nagaoka, "Making good codes for classical-quantum channel coding via quantum hypothesis testing," IEEE Trans. Inf. Theory 53, 2261-2266 (2007).

${ }^{123}$ T. M. Cover and J. A. Thomas, Elements of Information Theory, 2nd ed. (Wiley, 2006). 